\title{
An Innovative Decision Rule Approach to Tyre Pressure Monitoring
}

\author{
Oche Alexander Egaji ${ }^{\mathrm{a}, *}$, Salem Chakhar ${ }^{\mathrm{b}}$, David Brown ${ }^{\mathrm{c}}$ \\ ${ }^{a}$ Centre of Excellence in Mobile and Emerging Technologies (CEMET), Faculty of Computing, Engineering and Science, \\ University of South Wales, Pontypridd, UK. \\ ${ }^{b}$ Portsmouth Business School and Centre for Operational Research \& Logistics, University of Portsmouth, Portsmouth, UK. \\ ${ }^{c}$ Institute of Industrial Research, University of Portsmouth, Portsmouth, UK.
}

\begin{abstract}
Tyres are one of the most important safety components on a vehicle. Ignoring or failing to correctly set the tyre pressure may lead to accidents, and can affect the vehicle's fuel efficiency and tyre lifespan. Hence, there is a need for a Tyre Pressure Monitoring System (TPMS) that can effectively monitor tyre condition. The current threshold-based TPMSs are characterised by a high number of false alarms. This is mainly due to: (i) the non-static and complex relationship between tyre pressure and temperature; and, (ii) the measurement error of the pressure/temperature sensors that are used for data collection. In this paper, we propose an innovative decision rule-based approach to tyre monitoring. This approach relies on the Dominance-based Rough Set Approach (DRSA), which is a well-known multicriteria classification and preference learning method. The DRSA takes a decision table as an input and it generates a collection of if-then decision rules as an output. The complexity of pressure/temperature relationship is solved by fixing one of the parameters and then generating the decision rules based on the other parameter. The problem of false alarms is solved by a discretisation of the scale of the fixed parameter. Based on these solutions, we designed two types of analysis levels: pressure-oriented analysis and temperature-oriented analysis. The proposed approach has been validated and implemented within an important travelling company that operates in the South of England. The real-world tests showed that the proposed approach has improved the current system and has led to a substantial reduction of false alarms.
\end{abstract}

Keywords: Rough sets, dominance-based rough set approach, tyre pressure monitoring system, tyre pressure, tyre temperature

\section{Introduction}

Although tyres are one of the most important safety components on a vehicle, they are often ignored. However, a tyre's condition is critical for vehicle safety. According to Royal Automobile Club (2016), over 3 million motorists put their life at risk by never checking their tyre pressure or general tyre condition. The study in Royal Automobile Club (2016) reveals that one in eight vehicles on the UK roads have at least one illegal tyre. This was described as an alarming degree of neglect by the Automobile Association (which is a British motoring association that provides car insurance, motoring and safety advice, and other services). TyreSafe, in partnership with Highways England, surveyed

\footnotetext{
${ }^{*}$ Corresponding author

Email addresses: alexander.egaji@southwales.ac.uk (Oche Alexander Egaji), salem.chakhar@port.ac.uk (Salem Chakhar), david.browneport.ac.uk (David Brown)
} 
over 340,000 tyres as they were being replaced at 819 retail outlets across England, Northern Ireland, Scotland and Wales. Their survey reveled that the average proportion of illegal tyres at the point of replacement across the UK was more than 27\%. This means that one-in-four of the 35 million cars and light commercial vehicles on the UK roads are currently being driven with illegal tyres. Dangerous tyre condition, which includes under- or Over-inflated tyres, is the largest single contributor to accidents that result in casualty. About $6 \%$ of fatal motorway accidents are a result of the sudden failure of Under-inflated tyres (Royal Automobile Club, 2016).

More so, a smaller tyre deflection (17\%) can increase fuel consumption by $2 \%$ and decrease the tyre's lifespan by $25 \%$. Every year in the European Union, 20 million tons of fuel are wasted, 2 million tons of carbon-dioxide are generated and 200 million tyres are wasted before the end of their expected lifespan (Marton et al., 2014). Tyre safety is simply not an option but is a necessity. This has prompted the need for a Tyre Pressure Monitoring System (TPMS) (see Section 2) that monitors the internal tyre conditions using sensors and which alerts the user when the temperature or pressure crosses a set threshold.

This paper proposes an innovative decision rule-based approach to tyre monitoring that is based on data collected from one of the UK's South-West coach operators. This approach relies on the Dominance-based Rough Set Approach (DRSA), which is a well-known multicriteria classification and preference learning method (Greco et al., 2001, 2002; Słowiński et al., 2002, 2012). The DRSA takes a decision table as an input and it then generates a collection of if-then decision rules. The DRSA has some powerful characteristics that make it attractive in real-world decision problems (Chakhar et al., 2016). Among the main characteristics of the DRSA is the use of a learning set as an input to elicit and generalise the preferences of the decision makers, which minimises the cognitive effort required from them. Although the use of a learning set as an input has been adapted in several multicriteria classification methods, the main addition of the DRSA as compared to other multicriteria classification methods is its simplicity and its easily understandable if-then decision rules provided as an output, while other methods have no such straightforward interpretation (Blaszczyński et al., 2012).

The non-static/dynamic relationship between tyre pressure and temperature aswell as the measurement error of the sensors, the application of the DRSA using both the temperature and pressure values will lead to a high number of non-coherent and conflicting decision rules. In addition, using the current threshold-based approach may lead to false alarms. The author's proposed solutions to resolve these issues consist of: (i) fixing one of the parameters and generating the decision rules based on the other parameters; and, (ii) a discretisation of the scale of the fixed parameter. These two solutions resolve the issue related to the non-static/dynamic relationship between tyre pressure and temperature, subsequently preventing false alarms. Based on these solutions, we designed two types of analysis levels: (i) pressureoriented analysis; and, (ii) temperature-oriented analysis. The principle of these analysis levels is to discretise the fixed parameter values into a set of ordered ranges, then construct a decision table for each range, and then use the DRSA to infer a collection of decision rules. The generated decision rules need to be validated and refined by adding the implicit condition relative to the fixed parameter (temperature in the case of pressure-oriented analysis or pressure in the case of temperature-oriented analysis).

The proposed approach has been validated and implemented within an important travelling company that operates 
in the South of England. Our real-world tests showed that the proposed approach considerably improved the tyre monitoring system and led to a very substantial reduction of false alarms.

The rest of the paper is organised as follows. Section 2 introduces the TPMS, and it discusses the main approaches to design and implement them. Section 3 details the decision rule-based proposed approach to the TPMS. Section 4 comments on the system implementation and validation. Section 5 presents the case study. Section 6 provides a comparative study and validates the analysis approach with large datasets. Section 7 concludes the paper.

\section{Tyre maintenance and existing approaches to tyre pressure monitoring systems}

This section introduces the concept of tyre maintenance and it describes the various forms of wear caused by incorrect tyre inflation (Section 2.1). It also explains TPMS, which can be divided into two categories, namely: direct TPMS (dTPMS) and indirect TPMS (iTPMS) (2.2). Finally, the main shortcomings of the current approaches are discussed (Section 2.3).

\subsection{Tyre maintenance}

Ensuring that tyres are inflated at the correct pressure is vital because the pressurised air in the tyres helps to support the weight of the vehicle and its load. As mentioned in Section 1, it can also affect the vehicle's fuel efficiency. The required tyre pressure often varies among vehicle type, tyre make and/or load being carried. It is recommended that the tyre pressure is checked regularly against the manufacturer's recommended pressure for the right load profile of the vehicle. This is essential for commercial vehicles because of the variation of their load. The recommended tyre inflation pressure is normally based on the vehicle's specification and on the tyre's walls. The effects of having the correct or incorrect inflation pressure on the tyre wear/tread pattern is shown in Figure 1. It can be seen that the tyre tends to wear more on both sides when Under-inflated and it wears more at the centre when Over-inflated. These uneven wear patterns are a risk to the driver, including: reducing road traction, increasing stop time, increasing the risk of tyre blowout (which can be fatal in some cases), and they can lead to a potential police fine and 3 points on a driving license. These issues have increased the need for a TPMS that is able to monitor the tyre condition in real-time.

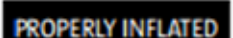

\section{UNDERINFLATED}
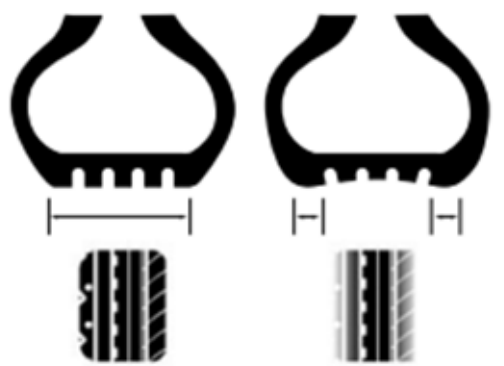

OVERINFLATED

Figure 1: Effect of Tyre Inflation Pressure

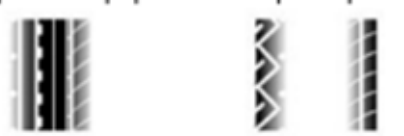

Source: http://www.rsa.ie/PageFiles/5206/TyreXSafety_Information_Guide_.PDF 


\subsection{Tyre pressure monitoring system}

A TPMS is an electronic system that is designed to monitor the air pressure inside pneumatic tyres. The TPMS reports the real-time tyre temperature and pressure reading to the driver or a central control unit. This can be done with a gauge, a pictogram display or a simple low-pressure warning light. There are two types of TPMS: dTPMS and iTPMS. The TPMS is provided either at factory level for cars or it can be made available as an aftermarket solution. The use of a TPMS can reduce the likelihood of road accidents caused by tyres, and they can increase fuel efficiency and reduce tyre wear due to Over- or Under-inflation.

\subsubsection{Indirect tyre pressure monitoring system}

An iTPMS relies on the wheel speed sensors that are used by the anti-lock braking system. These sensors measure the rate of revolution of each wheel, which are then compared to themselves and other vehicle operating data, such as the speed, through an on-board computer system. The first generation iTPMS are based on the premise that Underinflated tyres will have a smaller diameter (hence, a higher angular velocity) as compared to correctly inflated tyres. However, this approach fails when there are several Under-inflated tyres. The second generation iTPMS resolves the problem of simultaneous detection by using spectrum analysis of individual wheels. This can be realised using advanced signal processing techniques. Spectrum analysis is based on the principle that certain Eigen forms and frequencies of the tyre/wheel assemble are highly sensitive to the inflation pressure. These oscillations can be monitored through advanced signal processing of the wheel speed signals (Premarsha, 2016).

An example of an iTPMS is discussed in Na et al. (2016) (see also Na et al. (2017)). However, this approach of tyre monitoring can become inaccurate and unreliable when a different tyre is purchased, the tyres are unevenly worn, or the system had not been reset after proper inflation or after tyre rotation. Thus, tyre pressure must be measured directly to ensure that the driver/operator receives reliable information.

\subsubsection{Direct tyre pressure monitoring system}

A dTPMS employs pressure/temperature sensors on each wheel, either internal or external. The sensor measures the tyre pressure/temperature of the individual wheel and sends this information to the driver and/or to a data collection point. This device can detect Under-inflation in any combination, be it just one or multiple tyres simultaneously. This is presently the most widely used approach because it resolves some of the issues faced using iTMPS and can simultaneously detect tyres Under/Over-inflation. There are five types of dTPMS that are able to directly measure the tyre pressure/temperature of a heavy vehicle, which are: rim mount (inside tyre envelope), tyre patch (mounted to tyre inside tyre envelope), interior valve stem (inside tyre envelope), flow-through (outside of tyre) and end-of-valve stem mount (outside of tyre) (Premarsha, 2016). This paper used the rim mount dTPMS approach. The system consists of sensors, unique identifier chip, antenna, display unit, transceiver (trailer box) and vehicle identification number.

\subsection{Some existing approaches}

The current TPMS systems are pressure based and they are a threshold system, where the end user sets the minimum and maximum pressure for an alert. However, the tyre temperature and pressure relationship is not static since temperature varies with pressure in a complex way. This can result in a false alarm because the daily variation of tyre 
internal temperature can be considerably high, especially in a hot climate. This can cause the pressure to exceed the set threshold, thereby triggering a false fault alarm. It is imperative to dynamically determine the appropriate pressure at certain temperature to avoid a false alarm and also to detect early tyre defects. This can be resolved by an appropriate decision making algorithm that is temperature and pressure dependant.

Vishnoi et al. (2015) developed a wireless TPMS electronic system that measures the tyre's internal temperature/pressure and then sends this data through a Radio Frequency (RF) remote controlto an on-board system. This paper focused on the hardware requirements for the design of a simplified TPMS system with no data analysis. Hasan et al. (2011) introduced a method to implement TPMS in vehicles. These authors proposed a system that relies solely on air pressure inside pneumatic tyres of automobiles. A warning is generated whenever tyre pressure crosses the maximum or minimum safe pressure level, or when it changes abruptly. The lower level and upper limit of tyre pressure or safe range of abrupt change can be modified by the user. The paper by Karhe \& Patil (2016) focused on the design and development of a TPMS system based on Android using the ATmega16 microcontroller. The tyre temperature and pressure measurements from the sensors are displayed on an Liquid-Crystal Display (LCD) display and they can be sent to an Android phone through Bluetooth. There is no additional processing of the temperature and pressure measurements.

Garcia-Pozuelo et al. (2017) implemented a novel strain-based method to estimate tyre conditions such as inflation pressure, vertical load or rolling speed using fuzzy logic. Coppo et al. (2017) developed a multi-sensing set-up to measure experimentally the tyre strain using optical sensors on a rolling tyre. Kubba \& Jiang (2014) conducted a compressive study of TPMS technologies and potential energy saving solutions. The study focused on comparing various types of pressure transducers, their measuring accuracy and power consumptions.

Svensson et al. (2017) designed and developed an iTPMS using a supervised machine learning approach. The system permits to detect both incorrect tyre pressure and thread depth for different type of vehicles within a fleet without the need for additional physical sensors or vehicle specific parameters. The empirical results showed that the developed system has an accuracy of $90.54 \%$. The limitation of their work lies in the use of iTPM as outlined in Section 2.2.1. More so, their confusion matrix showed that $10.93 \%$ of the mislabelled are false negative and $89.9 \%$ are false positive. This means more faulty tyres are misclassified as good, which can be catastrophic. It is important that this false positive be reduced to the barest minimum.

The authors in $\mathrm{Li}$ et al. (2010) proposed a fuzzy comprehensive evaluation model to determine and classify tyre safety states into five levels of safety, namely: Very Safe, Safe, Basic Safe, Dangerous, Very Dangerous. The fuzzy model consists of five input variables, which are: tyre temperature, pressure, vehicle speed, tyre load and tyre wear. They used the Analytic Hierarchy Process (Saaty, 1980) to compute the relative weight of all the input parameters. These weights are then used to identify the tyre's safety state.

\subsection{Summary}

Based on this discussion, we can identify several shortcomings that characterise existing approaches:

- The current threshold-based TPMSs are characterised by a high number of false alarms. This is mainly due to: (i) 
the complex relationship between tyre temperature and pressure (which are the main input parameters to TPMS); and, (ii) the measurement errors of the sensors used for the data collection.

- Also, most of the existing studies focused on the technical aspects of the TPMS hardware, and they gave little attention to the decision aspects.

- Apart from the proposal of Li et al. (2010), none of the above-cited studies addresses tyre pressure monitoring using multicriteria analysis. Most of the previous systems focused on a single parameter (most often pressure) along with a set threshold.

- As a consequence, existing approaches cannot handle the complex relationship between pressure and temperature variables and their preference direction.

- The use of conventional machine learning approaches is insufficient to consider the ordinal relationships that characterise tyre health situations.

\section{Proposed approach}

The objective of this section is to present a rule-based decision approach to TPMS. This approach relies on the DRSA, which is a well-known multicriteria classification and preference learning method. A brief overview of the DRSA is provided in Section 3.1, and followed by an analysis strategy in Section 3.2. Induction and refinement of monitoring rules are addressed in Section 3.3. Finally, the validation and exploitation of these rules are discussed in Section 3.4.

\subsection{Principles of Dominance-based Rough Set Approach}

The extraction of monitoring rules relies on a well-known multicriteria classification method, namely the DRSA. The DRSA principles and concepts are given in the rest of this subsection and a formal and brief overview of DRSA is given in Appendix A. The input for the DRSA is a decision table $\mathbf{S}=\langle U, Q, V, f\rangle$, where $U$ is a non-empty finite set of objects and $Q$ is a non-empty finite set of attributes such that $q: U \rightarrow V_{q}$ for every $q \in Q$. The $V_{q}$ is the domain of attribute $q, V=\bigcap_{q \in Q} V_{q}$, and $f: U \times Q \rightarrow V$ is the information function defined such that $f(x, q) \in V_{q}$ for each attribute $q$ and object $x \in U$. The set $Q$ is divided into a subset $F \neq \emptyset$ of condition attributes and a subset $D \neq \emptyset$ of decision attributes, such that $F \cup D=Q$ and $F \cap D=\emptyset$. The domains of the condition attributes are supposed to be ordered according to a decreasing or increasing preference. These attributes are called criteria. The DRSA assumes that the preference is increasing with $f(\cdot, q)$ for every $q \in F$. They also assume that the set of decision attributes $D=\{E\}$ is a singleton. The unique decision attribute $E$ makes a partition of $U$ into a finite number of preference-ordered decision classes $\mathbf{C l}=\left\{C l_{t}, t \in T\right\}, T=\{0, \cdots, n\}$, such that each $x \in U$ belongs to one and only one class.

Due to the ordinal nature of decision classes, the knowledge is represented through a collection of upward unions $C l_{t}^{\geq}$and downward unions $C l_{t}^{\leq}$of classes of classes as follows:

$$
C l_{t}^{\geq}=\bigcup_{s \geq t} C l_{s}, C l_{t}^{\leq}=\bigcup_{s \leq t} C l_{s} .
$$




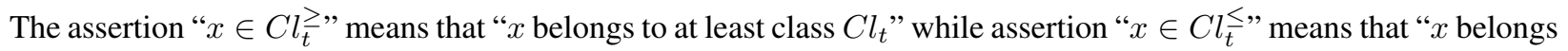
to at most class $C l_{t} "$.

The main philosophy of rough set theory is to handle inconsistent data by clearly separating certain and doubtful information. By inconsistent data, we mean data containing examples described by the same criteria values but assigned to different decision classes. This means that rough set theory does not correct or aggregate inconsistencies but handles them by determining for each decision class its lower approximation (corresponding to districts which certainly belong to the class according to their description) and its upper approximation (corresponding to districts which possibly belong to the class). Boundary is the difference between upper and lower approximations.

The definition and computation of the lower and upper approximations relies on the dominance relation. The principle of dominance requires that a district $x$ dominating district $y$ on all considered criteria (i.e. $x$ having evaluations at least as good as $y$ on all considered criteria) should also dominate $y$ on the decision (i.e. $x$ should be assigned to at least as good decision class as $y$ ). Districts satisfying the dominance principle are called consistent, and those violating the dominance principle are called inconsistent.

The dominance relation is used to define two types of knowledge granules generated by the criteria set. These granules are dominance cones associated with each district $x$ in the set of learning examples. The first type is denoted $\Delta^{+}(x)$ and called dominating set or positive dominance cone containing the district that dominates $x$. The second type is denoted $\Delta^{-}(x)$ and called the dominated set or negative cone containing the district dominated by $x$.

The lower approximation of union of classes $C l_{t}$ is composed of such districts $x$ that the positive dominance cone $\Delta^{+}(x)$ in the criteria space is included in this union. The upper approximation of union of classes $C l_{t}^{\geq}$is composed of such districts $x$ that the negative dominance cone $\Delta^{-}(x)$ in the criteria space has a non-empty intersection with this union. Analogously, the lower approximation of union of classes $C l_{t}^{\leq}$is composed of such districts $x$ that the negative dominance cone $\Delta^{-}(x)$ in the criteria space is included in this union. The upper approximation of union of classes $C l_{t}^{\leq}$is composed of such districts $x$ that the positive dominance cone $\Delta^{+}(x)$ in the criteria space has a non-empty intersection with this union.

The obtained approximations are then used to induce a collection of decision rules that summarize the content of decision table. Decision rules take the form of 'if-then' conditional statements. The condition part specifies the values assumed by one or more criteria, and the decision part specifies an assignment to one or more decision classes. The following three types of decision rules may be considered: (i) certain rules generated from the lower approximations of unions of classes; (ii) possible rules generated from the upper approximations of unions of classes; and, (iii) approximate rules generated from the boundary regions. The general structures of certain decision rules are as follows:

\section{IF condition(s), THEN At Most $C l_{t}$ \\ IF condition(s), THEN At Least $C l_{t}$}

The decision part of a certain decision rule takes the form of an assignment to at most class unions or at least class unions. The general structures of the possible decision rules is similar to those of certain decision rules but their decision parts are of the form "Possibly At Most Cl $l_{t}$ " or "Possibly At Least $\mathrm{Cl}_{t}$. Approximate decision rules have a decision part of the form "Belongs to $C l_{s} \cup C l_{s+1} \cup \cdots \cup C l_{t}$ ". 
An object supports a decision rule if the description of the object matches both the condition and the decision parts of this rule. The support of a rule is the number of objects supporting the rule. A decision rule covers an object if the description of the object matches at least the condition part of the rule. The coverage is the number of the object covered by the rule. The strength of a rule is the number of positive examples covered by the rule. The relative strength is the number of positive examples covered by the rule divided by the number of all positive examples in the union of classes. We note that if the consequence is univocal (i.e., contains only one decision), the rule is exact; otherwise, it is approximate.

\subsection{Analysis strategy}

The first investigations of the raw data related to tyre monitoring show that the relationship between temperature and pressure is not static as the temperature varies with pressure in a complex way. The raw data from one of the coaches that we used as a testbed with a tyre size $315 \backslash 80$ is shown in Figures 2 and 3. Figure 2 shows the time series of temperature and pressure measurements from a real-time data while Figure 3 shows the raw data temperature versus pressure plot. Based on these figures, the health of a tyre profile can be classified into three zones or categories: 0 Normal, 1 - Over-inflated and 2 - Under-inflated. Considering that a tyre size of $315 \backslash 8022.5$ was used for this paper; for a given temperature, the pressure can vary between $[100,160]$ and similarly for a given pressure, the temperature can also vary. An immediate consequence of this is that we cannot specify the preference direction of the considered criteria because the normal situation (i.e. zone 0) is somewhere between a high pressure value (i.e. in zone 2 ) and a low pressure value (i.e. in zone 1).

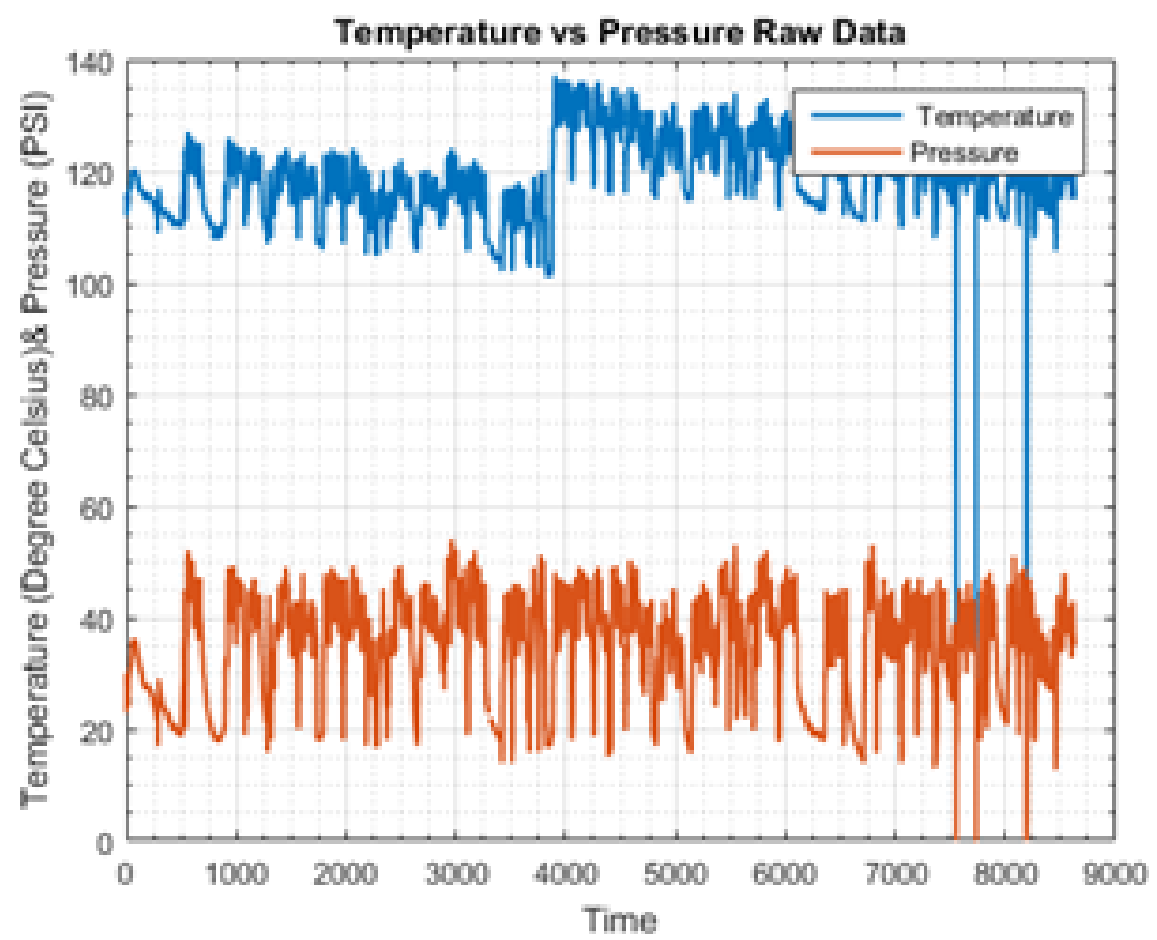

Figure 2: Temperature and pressure measurements from a real-time data 


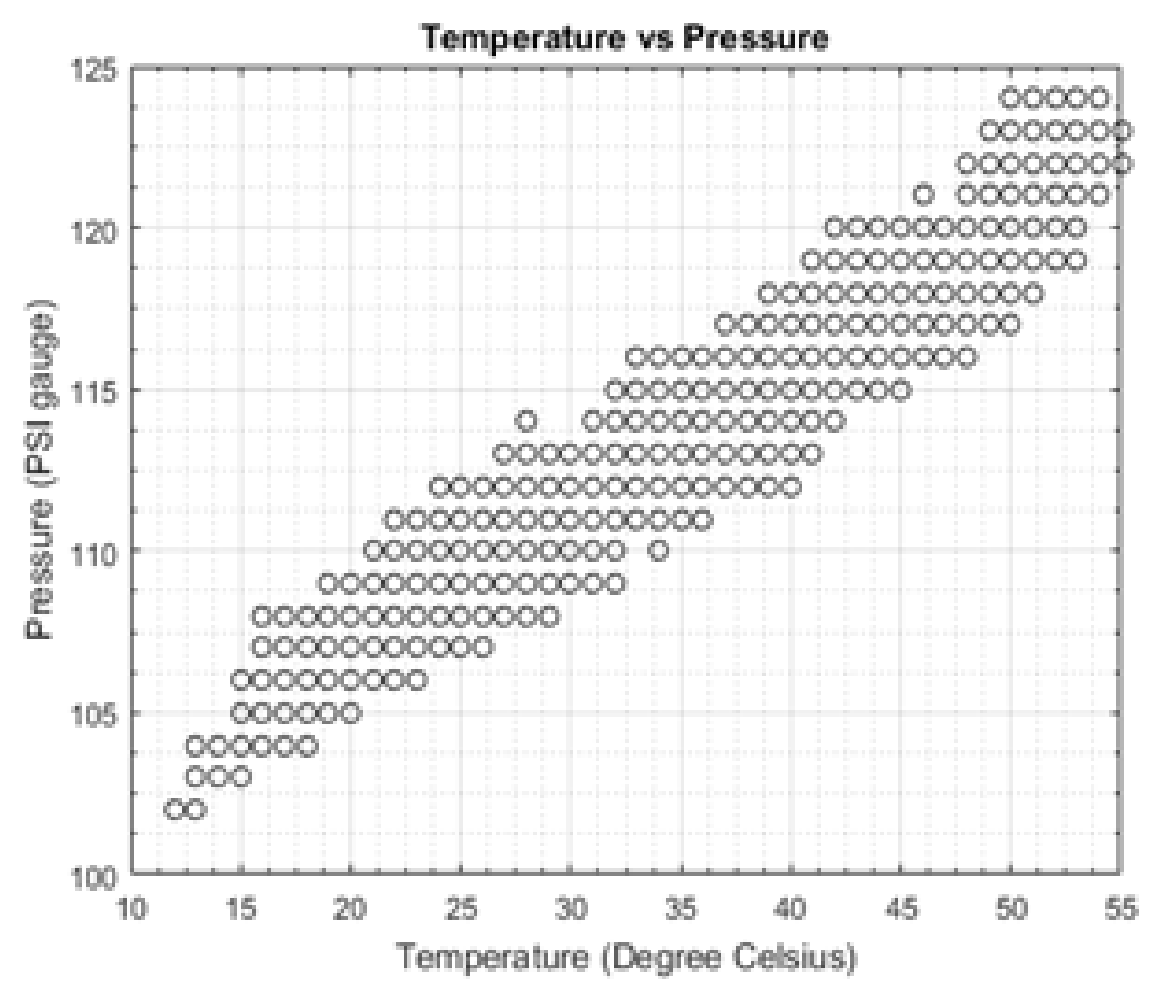

Figure 3: raw data temperature versus pressure

Due to the complex pressure/temperature relationship and measurement error, the application of DRSA using jointly the temperature and pressure values will lead to a high number of non-coherent and conflicting decision rules. To avoid the problem of pressure/temperature complex relationship, one possible solution consists of fixing one of the criteria and then generating the rules for the other criterion. Because both criteria are continuous, including all possible values, this will lead to a very high number of decision rules. Furthermore, due to the measurement error of the sensors, the decision rules will overlap. The idea that we have used in this paper to address this additional issue by relying on a discretisation of the scale of the fixed criterion to obtain an ordered series of intervals and maintain the values of the other criterion as they are. Two discretisation factors are required: $\alpha$ for pressure and $\beta$ for temperature.

The application of the DRSA requires the identification of a subset of learning examples and their assignment to a predefined set of decision classes. The decision objects in the considered case study correspond to the different readings collected by the different sensors. The three decision classes $C l_{0}, C l_{1}$ and $C l_{2}$ that are considered in this paper correspond to the three zones, $0-1-2$. These decision classes obey the following preference order $C l_{0} \prec C l_{1} \prec C l_{2}$, which can be justified as follows. Decision class $C l_{0}$ corresponds to the Normal condition and there is no action to handle. Decision class $C l_{1}$ corresponds to Over-inflation, which could lead to a reduced traction uneven wear on the centre of the centre. Decision class $\mathrm{Cl}_{2}$ corresponds to Under-inflation, which can be more dangerous. When tyres are Under-inflated, the internal temperature increases, which can overheat the building structure of the tyres. This can cause a tyre blowout, which can be fatal. Additionally, Under-inflated tyres are not economical because they increase 
the fuel consumption of a vehicle due to their increased rolling resistance.

Based on this discussion, we designed an analysis strategy that is illustrated graphically in Figure 4. Two main analysis levels can be distinguished: (i) pressure-oriented analysis; and, (ii) temperature-oriented analysis. Each of these analysis levels will be further subdivided into two sub-levels and only two decision classes are considered: the first sub-level analysis uses the decision classes $C l_{0}$, and $C l_{1}$ and the second sub-level uses the decision classes $C l_{0}$ and $C l_{2}$. This will lead to four analysis levels, as illustrated in Figure 4 . The pressure-oriented analysis starts by a discretisation of the temperature values into collection of ranges $T$ and for each range $T_{k} \in T$ (where $k=i, i+\alpha, i+2 \alpha, \ldots, n$; $\alpha$ is the width of ranges, and $n$ is the number of ranges) constructs two decision tables $\mathbf{S}_{k}$ and $\mathbf{S}_{k}^{\prime}$ each with one condition criterion relative to tyre pressure and one decision attribute relative to the health of the tyre. The decision table $\mathbf{S}_{k}$ includes the readings relative to Under-inflation $\left(\mathrm{Cl}_{1}\right)$ and Normal $\left(\mathrm{Cl}_{0}\right)$ conditions, where the Pressure criterion is specified as cost. The decision table $\mathbf{S}_{k}^{\prime}$ includes the readings relative to Over-inflation $\left(\mathrm{Cl}_{2}\right)$ and Normal $\left(\mathrm{Cl}_{0}\right)$ situations, where the Pressure criterion is specified as gain.

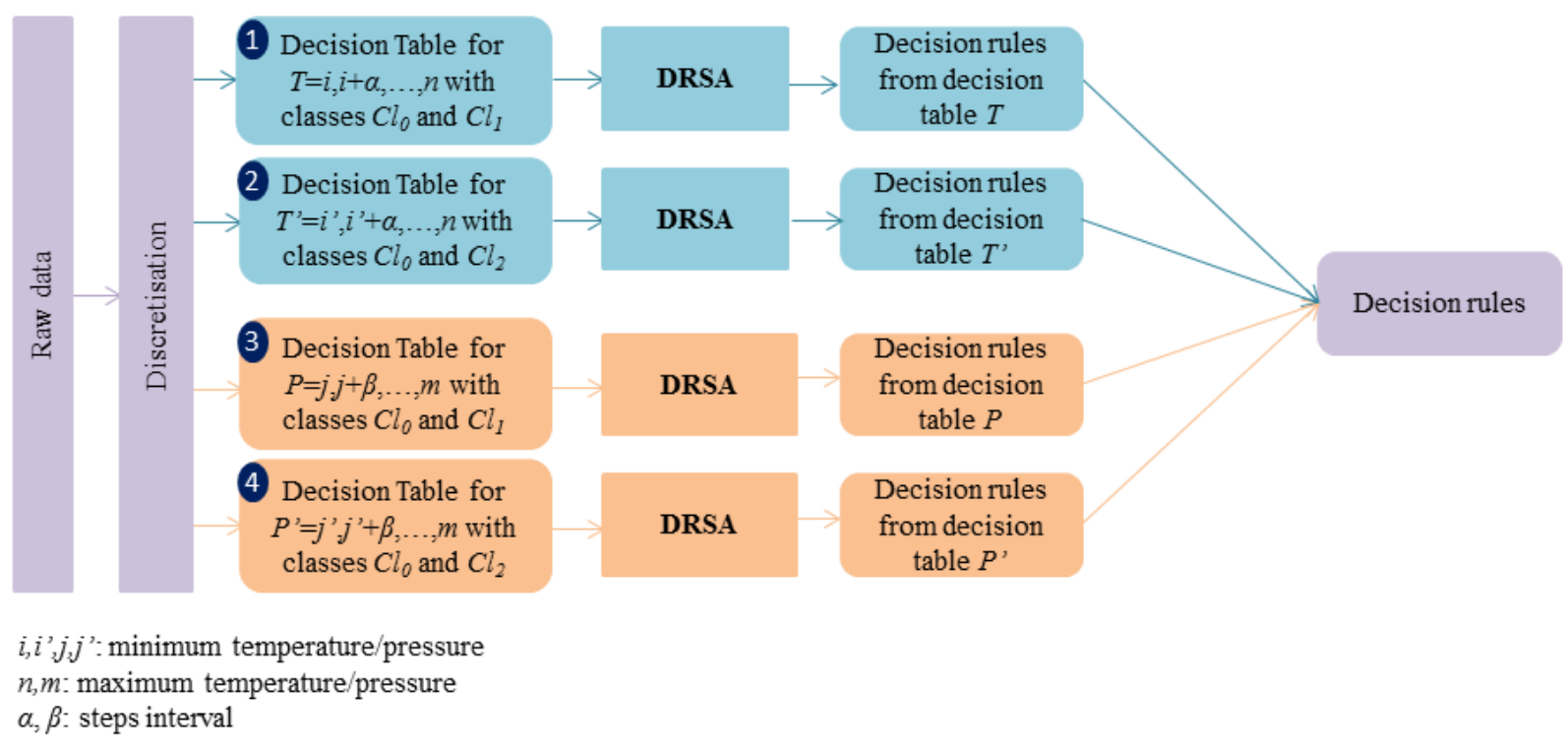

Figure 4: Analysis strategy

Similarly, the temperature-oriented analysis starts by a discretisation of the pressure values into collection of ranges $P$ and for each range $P_{h} \in P$ (where $h=j, j+\beta, j+2 \beta, \ldots, m ; \beta$ is the width of ranges, and $m$ is the number of ranges) constructs two decision tables $\mathbf{S}_{h}$ and $\mathbf{S}_{h}^{\prime}$, each with one condition criterion relative to temperature and one decision attribute relative to the health of the tyre. The decision table $\mathbf{S}_{h}$ includes the readings relative to Under-inflation $\left(C l_{1}\right)$ and Normal $\left(C l_{0}\right)$ conditions, where the Temperature criterion is specified as cost. The decision table $\mathbf{S}_{h}^{\prime}$ in turn includes the readings relative to Over-inflation $\left(\mathrm{Cl}_{2}\right)$ and Normal $\left(\mathrm{Cl}_{0}\right)$ conditions, where the Temperature criterion is specified as gain.

After the construction of the decision tables, the DRSA can then be used to approximate the decision classes' unions as explained in Section 3.1 by using the equations given in Appendix A. In this analysis strategy, the decision tables 
used as input to DRSA use two decision classes $\left(C l_{0}\right.$ and $C l_{1}$ for the first and third analysis levels; or $C l_{0}$ and $C l_{2}$ for the second and fourth analysis levels) with two different preferences orders $C l_{0} \prec C l_{1}$ (for the first and third analysis levels) or $C l_{0} \prec C l_{2}$ (for the second and fourth analysis levels). In addition, the non-fixed criterion for both analyses levels is specified as gain.

\subsection{Induction and refinement of monitoring rules}

The monitoring rules are extracted from the rough approximations obtained by applying the DRSA. More details on rules induction are given in Appendix A.3. As mentioned earlier, three types of decision rules can be considered for the application purposes of DRSA: certain, possible and approximate. The monitoring rules considered in this application rely on certain decision rules. This choice is not specific to the application considered in this paper since possible and approximate decision rules are often used only for sensitivity analysis or when there is no or very few certain decision rules.

The decision part of a certain decision rule takes the form of an assignment to at most class unions or at least class unions. The first investigations show that rules obtained in the case of the first sub-level of pressure-oriented analysis have the following general syntaxes:

- If Pressure $\geq p_{2}$ then Health $=$ At most $C l_{0}$ (Normal),

- If Pressure $\leq p_{1}$ then Health $=$ At least $C l_{1}$ (Under-inflation),

and the rules obtained in the case of the second sub-level of pressure-oriented analysis have the following general syntaxes:

- If pressure $\leq p_{3}$ then Health $=$ At most $C l_{0}$ (Normal),

- If Pressure $\geq p_{4}$ then Health $=$ At least $C l_{2}$ (Over-inflation),

where $p_{1}, p_{2}, p_{3}$ and $p_{4}$ are the pressure values that verify $p_{1}<p_{2}<p_{3}<p_{4}$. All of the decision rules are certain (because they indicate a single decision class). The four decision rules are illustrated graphically in Figure 5(a). Figure 5(a) shows that these four rules can be replaced by three decision rules, as shown below:

- If Pressure $\geq p_{2}$ and Pressure $\leq p_{3}$ then Health $=C l_{0}$ (Normal)

- If Pressure $\leq p_{1}$ then Health $=$ Health $=C l_{1}$ (Under-inflation)

- If Pressure $\geq p_{4}$ then Health $=$ Health $=C l_{2}$ (Over-inflation)

A careful examination of Figure 5(a) indicates that the decision parts for 'Pressure $\geq p_{4}$ ', 'Pressure $\leq p_{1}$ ' and ' $p_{2} \leq$ Pressure $\leq p_{3}$ ' is straightforward. It can also be noted that the gaps between $p_{1}$ and $p_{2}$, and between $p_{3}$ and $p_{4}$ are due to discretisation. Hence, $p_{1}+\alpha=p_{2}$ and $p_{3}+\alpha=p_{4}$ where $\alpha$ is the discretisation factor and it is equivalent to the measurement error. The decision rules have been inferred for a given temperature range $T_{k} \in T$. Thus, they should be transformed by adding the implicit condition relative to the temperature. The final list of rules for range $T_{k}$ will be as follows: 


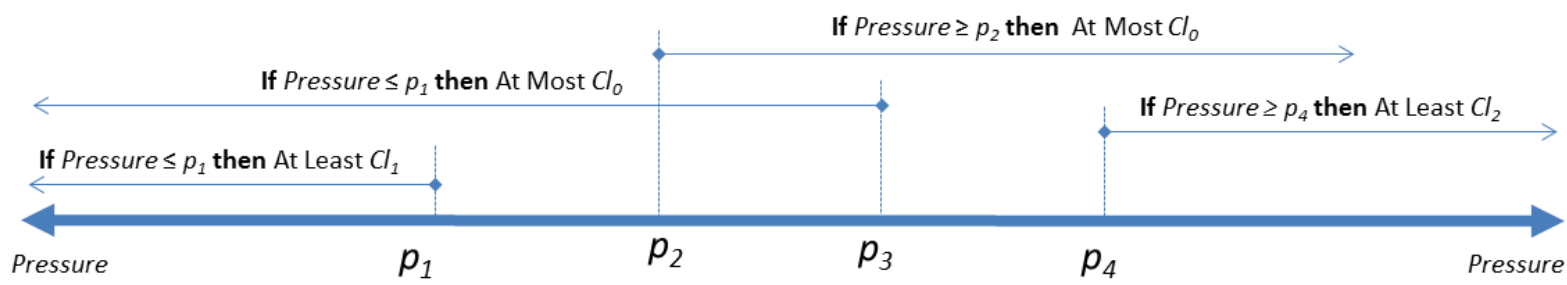

(a)

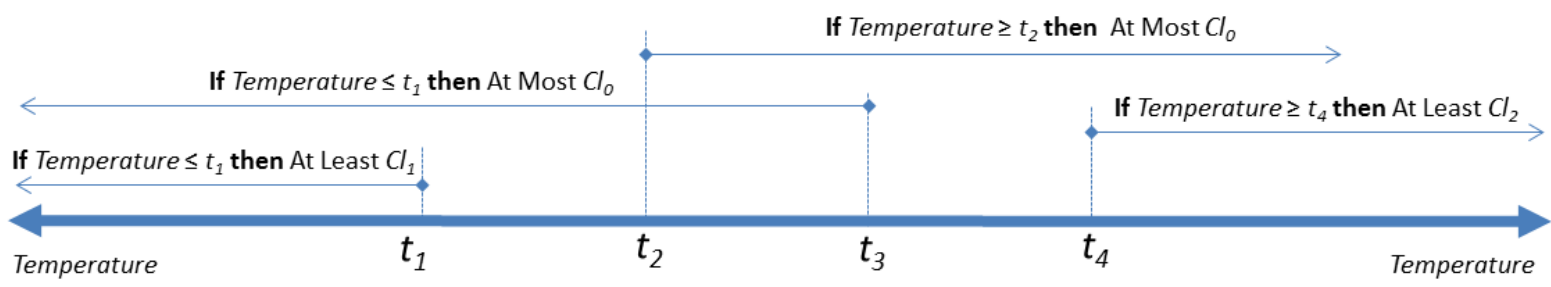

(b)

Figure 5: Overlapping of decision rules

- If Temperature $\in T_{k}$ and Pressure $>p_{1}$ and Pressure $<p_{4}$ then Health $=C l_{0}$ (Normal)

- If Temperature $\in T_{k}$ and Pressure $\leq p_{1}$ then Health $=C l_{1}$ (Under-inflation)

- If Temperature $\in T_{k}$ and Pressure $\geq p_{4}$ then Health $=C l_{2}$ (Over-inflation).

Similarly, the decision rules obtained in the case of the first and second sub-levels of temperature-oriented analysis have the following general syntaxes:

- If Temperature $\geq t_{2}$ then Health $=$ At most $C l_{0}$ (Normal),

- If Temperature $\leq t_{1}$ then Health $=$ At least $C l_{1}$ (Under-inflation),

- If Temperature $\leq t_{3}$ then Health $=$ At most $C l_{0}$ (Normal),

- If Temperature $\geq t_{4}$ then Health $=$ At least $C l_{2}$ (Over-inflation),

where $t_{1}, t_{2}, t_{3}$ and $t_{4}$ are the temperature values that verify $t_{1}<t_{2}<t_{3}<t_{4}$. All of the decision rules are certain (because they indicate a single decision class). These rules are illustrated graphically in Figure 5(b). The four rules in Figure 5(b) can then be replaced by the following three decision rules:

- If Temperature $\geq t_{2}$ and pressure $\leq t_{3}$ then Health $=C l_{0}$ (Normal)

- If Temperature $\leq t_{1}$ then Health $=C l_{1}$ (Under-inflation) 
- If Temperature $\geq t_{4}$ then Health $=C l_{2}$ (Over-inflation).

The analysis of the condition of the decision rules show that $t_{1}+\beta=t_{2}$ and $t_{3}+\beta=t_{4}$ where $\beta$ is the discretisation factor that is equivalent to the measurement error. Finally, the decision rules have been inferred for a given pressure range $P_{h} \in P$. Thus, they should be transformed by adding the implicit condition relative to the pressure. The final list of rules for the range $P_{h}$ will be as follows:

- If Pressure $\in P_{h}$ and Temperature $>t_{1}$ and Temperature $<t_{4}$ then Health=Cl $l_{0}$ (Normal)

- If Pressure $\in P_{h}$ and Temperature $\leq t_{1}$ then Health $=C l_{1}$ (Under-inflation)

- If Pressure $\in P_{h}$ and Temperature $\geq t_{4}$ then Health $=C l_{2}$ (Over-inflation).

\subsection{Validation and exploitation of monitoring rules}

The generated monitoring rules first need to be validated and they can then be used in practice. The authors in Hu et al. (2017) distinguish three complementary techniques for decision rules validation: (i) direct analysis of the monitoring rules analysis; (ii) reclassification; and, (iii) cross-validation. In the direct analysis validation technique, the decision maker is asked to consider all the decision rules and indicate his/her level of agreement on a five-level Likert scale (strongly disagree to strongly agree). When required, the decision maker can suggest modifications to one or more monitoring rules.

The reclassification analysis is based on the use of the generated monitoring rules on the original data. In theory, the application of these rules should lead to the same health status as the tyres in the original data. In practice, however, especially with real industrial data, this is not always the case and a limited number of misclassifications may be tolerated. If the number of misclassifications becomes unacceptable, then the learning phase must be iterated and the decision maker asked to revise his/her initial assignments to improve the quality of the monitoring rules generated.

In the cross-validation analysis, the available data is divided into training and testing subsets, and they are then used to evaluate the prediction accuracy of the system. The training subset trains the model and the testing subset measures the accuracy of the prediction. Several iterations of cross-validation are performed on different subsets of training and testing data, and the validation results are averaged over the rounds. The key difference between cross-validation and reclassification is that the testing data subset is always unknown and not used to train the system previously.

In this paper, the reclassification technique is used to validate the initial set of monitoring rules (see Section 5.3.1 and Section 5.3.2).

\section{System implementation and validation}

The obtained and validated decision rules have been used to design a classifier that will be embedded, through a series of plugins, in the TPMS. In this section, the architecture of the monitoring system is discussed (Section 4.1) and a certain number of meta-rules were developed to enhance the tyre monitoring system (Section 4.2). 


\subsection{Monitoring system architecture}

The network architecture for the system ${ }^{1}$ is shown in Figure 6. The modem enables the connection of various remote application devices via cellular network. It provides local control, connection management and key performance data. The modem consists of an ARM Cortex M4 32-bit processor with recognised interfaces, which allows the users to develop custom software programs to monitor and control application devices. The modem range covers $2 \mathrm{G}, 3 \mathrm{G}$ and 4G families. The communication ports are USB and RS232, and the modem uses a Subscriber Identification Module (SIM) card to connect to the mobile network to transmit tyre information and location to a central point/server. The modem is powered by the vehicle's $12 \mathrm{v}$ power supply, it is located under the vehicle's dashboard and it is hard wired to the vehicle display unit. It has a magnetic dashboard mounted antenna unit, which used by the receiver for the GPS signals and by the transmitter to transmit tyre information and position via the mobile telephone network.

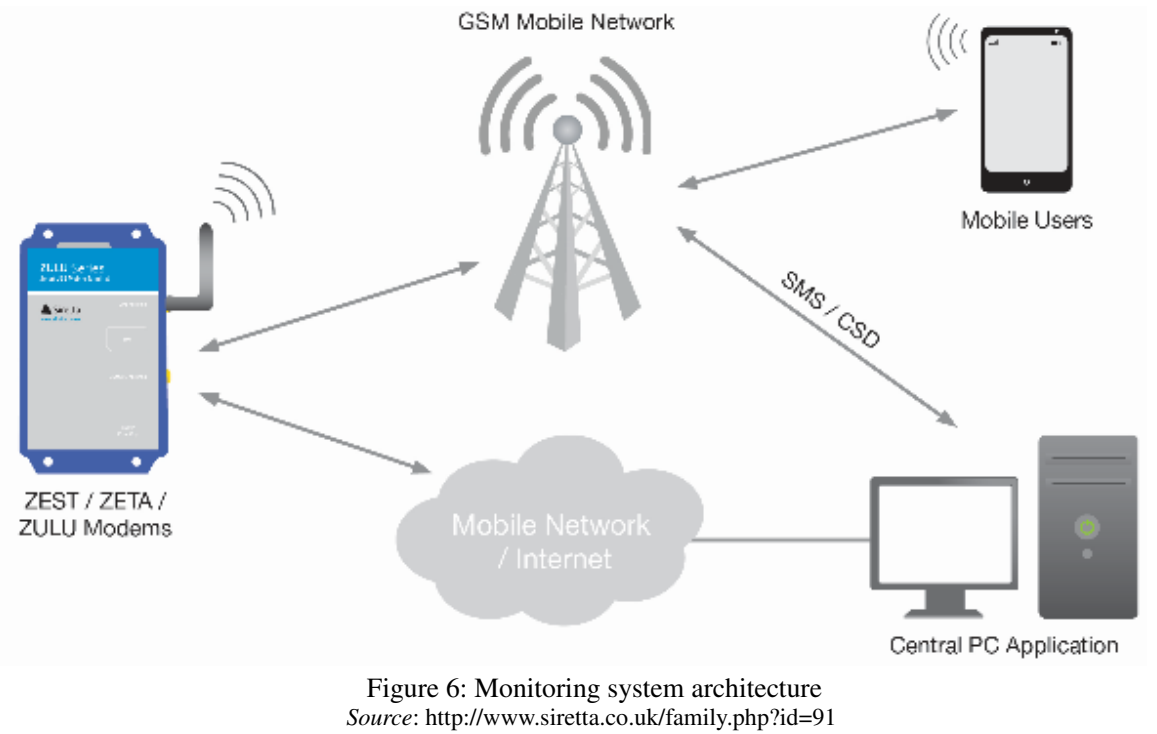

A data flow schematic for the system is shown in Figure 7. The sensors measure the tyre temperature and pressure readings; this information can be monitored by the driver through the display unit. These data, including the tyre's information and location, are sent to a central control centre through a mobile network for further processing.

The decision rules have been used to construct the system classifier. The classifier takes as input the pressure $(P)$ and temperature ( $T$ ) measures, and it generates two decision variables: (1) $H_{P}$, which indicates the tyre health as specified by the pressure-based decision rules; and, (2) $H_{T}$, which indicates the tyre health as specified by the temperature-based decision rules. Both $H_{P}$ and $H_{T}$ can take one of three values: (i) 0 (tyre is under normal situation); (ii) 1 (tyre is under-inflated); or, (iii) 2 (tyre is over-inflated).

The classifier can be implemented as a collection of plugins and incorporated both at the level of the server and at the level of the vehicle. The version installed at the vehicle level will permit continuous monitoring even when there is communication problems between the vehicle and the central server.

\footnotetext{
${ }^{1}$ More information on the system architecture is available at http://www. siretta.co.uk/family.php?id=91.
} 


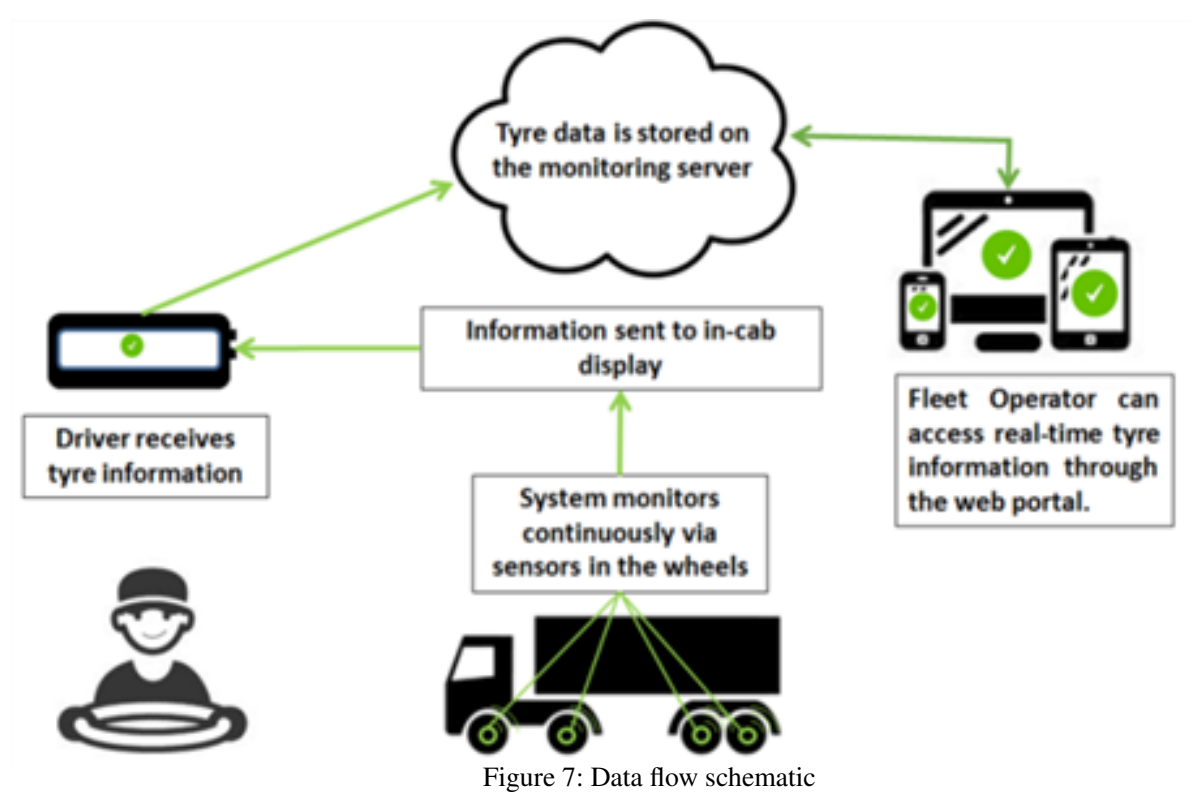

\subsection{Designing of monitoring meta-rules}

Using the rules obtained from the pressure-oriented analysis alone or those generated from the temperature-oriented analysis alone is sufficient to monitor the tyre health. However, it is better to include both rules from the temperature and pressure analysis levels to improve the trustworthiness of the system. The inputs of the monitoring system are the measured pressure $P$ and temperature $T$. However, in cases of data transition problems or a failure in the sensors, one or both of the input parameters $P$ and $T$ will be unknown (unk). There are four possible situations: (1) $P=u n k$ and $T=u n k$; (2) $P \neq u n k$ and $T=u n k$; (3) $P=u n k$ and $T \neq u n k$; and, (4) $P \neq u n k$ and $T \neq u n k$. The meta-rules that are associated with each of these situations will be explained in the rest of this section, as illustrated graphically in Figure 8. For situations (1) to (3), the measured pressure and/or temperature measures are unknown. In these situations, the monitoring system should send a message alerting the driver and control centre of the failed readings.

In the fourth case $(P \neq u n k$ and $T \neq u n k)$ both pressure and temperature measures are available. Two subcases can be distinguished here. The first subcase holds when the temperature-based health $\left(H_{T}\right)$ and the pressure-based health $\left(H_{P}\right)$ are equal. Three exclusive actions are possible here: (i) if $H_{T}=H_{T}=0$, then send a 'Tyre Temperature and Pressure are fine' info message; (ii) if $H_{T}=H_{T}=1$, then send a 'Tyre Under-inflated' alert; or, (iii) if $H_{T}=H_{T}=2$, then send a 'Tyre Over-inflated' alert. The second subcase holds when the temperature-based health $\left(H_{T}\right)$ and the pressure-based health $\left(H_{P}\right)$ are different. In this situation, if both $H_{T}>0$ and $H_{P}>0$, then the monitoring system should send a 'Tyre Temperature and Pressure are not fine' alert to the driver; otherwise, the monitoring system should send 'Tyre Temperature or Pressure are not fine' info message to driver.

\section{Case study}

This section presents a description of the company that we considered (Section 5.1), the dataset and how they were collected (Section 5.2) and it also discusses the application of the proposed approach (Section 5.3). 


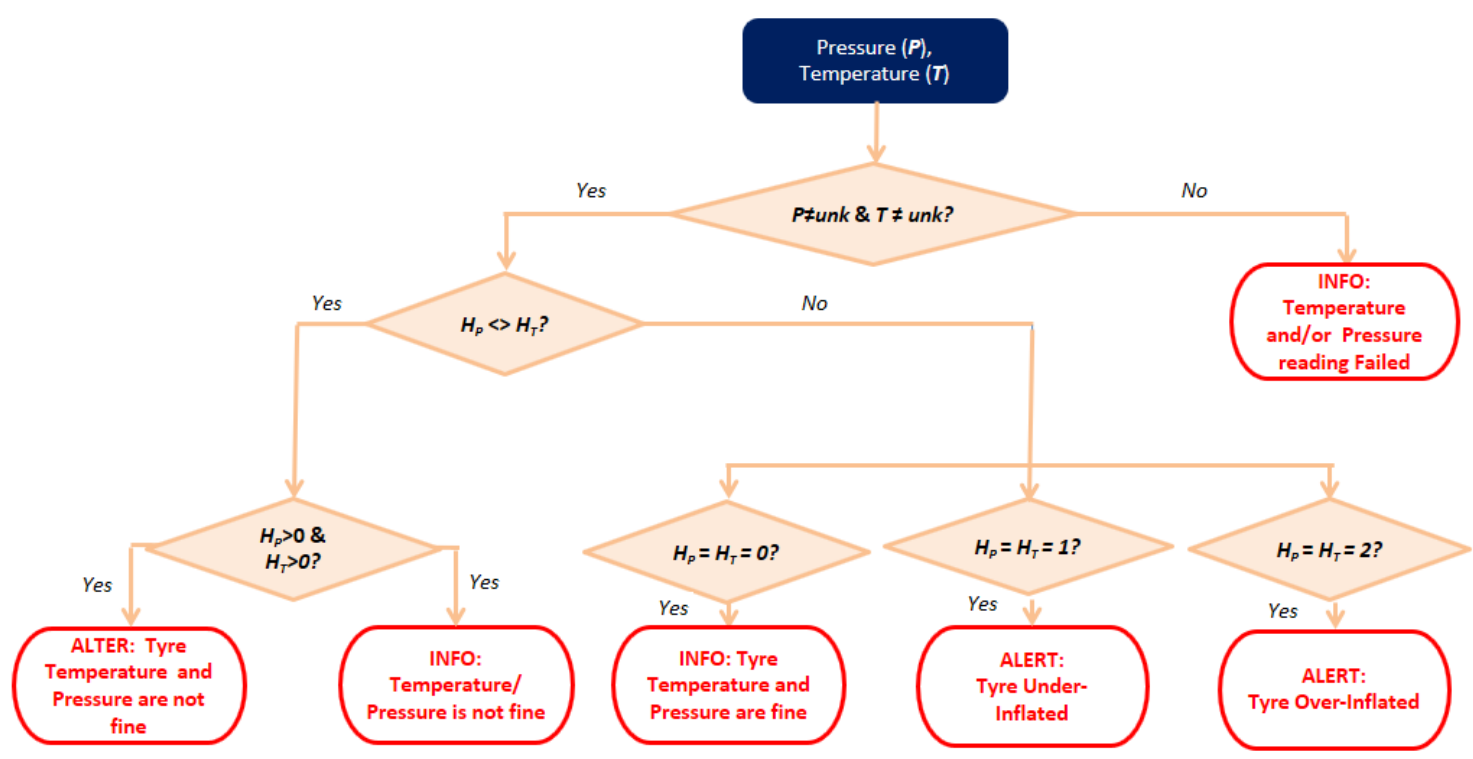

Figure 8: Working principles of meta-rules

\subsection{Company}

The proposed approach has been applied to data collected from one of the leading coach travel operators in the South of England. They operate several routes in the South of England on behalf of a leading national franchise passenger carrying operation. They run different prestigious and busy services from Gatwick Airport to London Victoria, and from Brighton to London. They also run three other services: from Portsmouth to Heathrow and Victoria, and from Southsea to Bristol route.

In this application, three of the group's coaches were used. They were fitted with three separate stages of tyre wear (all within legal requirements). The first coach was fitted with new tyres, the second was fitted with half worn tyres while the third was fitted with two thirds worn tyres. Each coach has an approximate run time of 18 hours a day, they only stop briefly to pick up passengers or change drivers. The data from the coaches are sent to a central database for further processing.

\subsection{Dataset}

The dataset was collected over a period of 6 months. For the purposes of this paper, only a subset of data has been used, which consists of tyre pressure, temperature, reading date/time and wheel position because these are the important components. Table 1 presents an extract from the dataset. The data were classified into three classes, as explained in Section 3.2. Each coach has six wheels, two at the front (1A,1D) and four at the rear (3A,3B,3C and 3D). A wireless sensor with a unique sensor ID is attached to each wheel, which measures the temperature and pressure of the tyre and then sends the data to a collection box (modem) located on the coach. This is explained in detail in section 4.1.

\subsection{Application and results}

The input to the DRSA is a decision table that contains a subset of readings concerning tyre pressure and temperature criteria. A description of these two criteria is given in Table 2. As shown in this table, both criteria are continuous. 
Table 1: Extract form collected raw data

\begin{tabular}{|c|c|c|c|}
\hline Tyre Pressure & Tyre Temperature & Reading Data/Time & Wheel position \\
\hline 116 & 30 & $13: 00.0$ & $1 \mathrm{~A}$ \\
\hline 112 & 23 & $15: 00.0$ & $1 \mathrm{~A}$ \\
\hline 113 & 23 & $13: 00.0$ & $1 \mathrm{~A}$ \\
\hline 113 & 24 & $36: 00.0$ & $1 \mathrm{~A}$ \\
\hline 113 & 24 & $40: 00.0$ & $1 \mathrm{~A}$ \\
\hline 113 & 24 & $44: 00.0$ & $1 \mathrm{~A}$ \\
\hline 113 & 24 & $47: 00.0$ & $1 \mathrm{~A}$ \\
\hline 122 & 30 & $13: 00.0$ & 1D \\
\hline 119 & 24 & $15: 00.0$ & 1D \\
\hline 118 & 24 & 13:00.0 & 1D \\
\hline 120 & 25 & $36: 00.0$ & 1D \\
\hline 118 & 24 & 03:00.0 & $1 \mathrm{D}$ \\
\hline 118 & 24 & 09:00.0 & $1 \mathrm{D}$ \\
\hline 119 & 25 & $12: 00.0$ & 1D \\
\hline 111 & 31 & $13: 00.0$ & $3 \mathrm{~A}$ \\
\hline 107 & 22 & $15: 00.0$ & $3 \mathrm{~A}$ \\
\hline 108 & 22 & $13: 00.0$ & $3 \mathrm{~A}$ \\
\hline 108 & 22 & $36: 00.0$ & $3 \mathrm{~A}$ \\
\hline 108 & 23 & $56: 00.0$ & $3 \mathrm{~A}$ \\
\hline 108 & 23 & 08:00.0 & $3 \mathrm{~A}$ \\
\hline 108 & 24 & $11: 00.0$ & $3 \mathrm{~A}$ \\
\hline 108 & 23 & $14: 00.0$ & $3 \mathrm{~A}$ \\
\hline 108 & 23 & $16: 00.0$ & $3 \mathrm{~A}$ \\
\hline 112 & 31 & $13: 00.0$ & $3 B$ \\
\hline 109 & 21 & $15: 00.0$ & $3 B$ \\
\hline 108 & 22 & $13: 00.0$ & $3 B$ \\
\hline 109 & 23 & $36: 00.0$ & $3 B$ \\
\hline 109 & 23 & 53:00.0 & $3 B$ \\
\hline 110 & 24 & 08:00.0 & $3 B$ \\
\hline 110 & 23 & $14: 00.0$ & $3 B$ \\
\hline 111 & 31 & $13: 00.0$ & $3 C$ \\
\hline 108 & 22 & $36: 00.0$ & $3 C$ \\
\hline 108 & 23 & $56: 00.0$ & $3 C$ \\
\hline 108 & 23 & 08:00.0 & $3 C$ \\
\hline 108 & 23 & $11: 00.0$ & $3 C$ \\
\hline 108 & 23 & $58: 00.0$ & $3 C$ \\
\hline 108 & 24 & 03:00.0 & $3 C$ \\
\hline 110 & 30 & $13: 00.0$ & $3 \mathrm{D}$ \\
\hline
\end{tabular}

The Pressure criterion values ranges from 100 to 169 while the Temperature criterion values ranges from 0 to 100 . The preferences of the criteria will vary according to analysis levels in Figure 4.

Table 2: Characteristics of used condition and decision attributes

\begin{tabular}{|c|c|c|c|c|c|c|c|}
\hline \multirow{2}{*}{$\begin{array}{c}\text { Analysis } \\
\text { Level }\end{array}$} & \multirow[t]{2}{*}{ Name } & \multirow[t]{2}{*}{ Description } & \multicolumn{2}{|l|}{ Preference direction } & \multirow[t]{2}{*}{ Data type } & \multirow[t]{2}{*}{ Possible values } & \multirow[t]{2}{*}{ Type } \\
\hline & & & Pressure oriented analysis & Temperature oriented analysis & & & \\
\hline $\begin{array}{l}\text { Level } 1 \& \text { Level } 3 \\
\text { in Figure } 4\end{array}$ & $\begin{array}{c}\text { Pressure } \\
\text { Temperature } \\
\text { Health }\end{array}$ & $\begin{array}{l}\text { Pressure of the tyre } \\
\text { Temperature of the tyre } \\
\text { Health of the tyre }\end{array}$ & $\begin{array}{l}\text { Cost } \\
\text { Gain } \\
\text { Gain }\end{array}$ & $\begin{array}{l}\text { Cost } \\
\text { Gain } \\
\text { Gain }\end{array}$ & $\begin{array}{c}\text { Continuous } \\
\text { Continuous } \\
\text { Ordinal }\end{array}$ & $\begin{array}{c}100 \text { to } 169 \\
0 \text { to } 100 \\
0,1\end{array}$ & $\begin{array}{l}\text { Condition } \\
\text { Condition } \\
\text { Decision }\end{array}$ \\
\hline $\begin{array}{c}\text { Level } 2 \text { \& Level } 4 \\
\text { in Figure } 4\end{array}$ & $\begin{array}{c}\text { Pressure } \\
\text { Temperature } \\
\text { Health }\end{array}$ & $\begin{array}{l}\text { Pressure of the tyre } \\
\text { Temperature of the tyre } \\
\text { Health of the tyre }\end{array}$ & $\begin{array}{l}\text { Gain } \\
\text { Gain } \\
\text { Gain }\end{array}$ & $\begin{array}{l}\text { Gain } \\
\text { Cost } \\
\text { Gain }\end{array}$ & $\begin{array}{c}\text { Continuous } \\
\text { Continuous } \\
\text { Ordinal }\end{array}$ & $\begin{array}{l}100 \text { to } 169 \\
0 \text { to } 100 \\
0,2\end{array}$ & $\begin{array}{l}\text { Condition } \\
\text { Condition } \\
\text { Decision }\end{array}$ \\
\hline
\end{tabular}

The values of the discretisation factors $\alpha$ and $\beta$ introduced in Section 3.2 depend on the sensors used for data collection. The Piezoresistive sensors used in this particular application have an operating temperature range of $-40^{\circ} \mathrm{C}$ to $85^{\circ} \mathrm{C}\left(-40^{\circ} \mathrm{F}\right.$ to $\left.185^{\circ} \mathrm{F}\right)$, a pressure accuracy of $\pm 0.25 \mathrm{Bar} / \pm 3.6 \mathrm{PSI}\left(\right.$ at $\left.0^{\circ} \mathrm{C} \sim 50^{\circ} \mathrm{C}\right)$ and a temperature accuracy of $\pm 3^{\circ} \mathrm{C} / \pm 5^{\circ} \mathrm{F}$ (at $0^{\circ} \mathrm{C} \sim 50^{\circ} \mathrm{C}$ ). Hence, the discretisation factors for pressure and temperature have been set to $\alpha=3$ and $\beta=2$, respectively. These values have been specified to be slightly less than the sensor accuracy ranges, namely \pm 3.6 PSI for pressure and $\pm 3^{\circ} \mathrm{C}$ for temperature). A series of preliminary tests confirm the validity of these values.

In this case study, and as discussed in Section 3.2, two levels of analysis have been distinguished: (i) pressureoriented analysis; and, (ii) temperature-oriented analysis. The result of the application of the DRSA in the first and second analyses is detailed in Section 5.3.1 and Section 5.3.2, respectively. We used the 4eMka2 (Greco et al., 1999) 
software, which implements the DRSA, in this case study.

\subsubsection{Pressure-oriented analysis}

The pressure-oriented analysis is based on a discretisation of the temperature criterion and then for each temperature value we define a decision table composed of two criteria corresponding to Pressure and Temperature and one decision attribute corresponding to the tyre's health. The values of Temperature criterion vary in the range $[0,100]$. Hence, a collection of 19 initial decision tables have been constructed. Table 3 provides the decision tables corresponding to temperature in the range $T_{9}$ which covers the temperature values in $[9,11]$ and the range $T_{30}$ which covers the temperature values in $[30,33]$.

Two different analyses have been conducted for each range: one uses the classes $C l_{0}$ and $C l_{1}$, and the other uses the classes $C l_{0}$ and $\mathrm{Cl}_{2}$. The first analysis corresponds to Level 1 in Figure 4. As indicated in Table 2 (in the fourth column), the criteria Pressure and Temperature are specified as cost-type and gain-type, respectively. In addition, the domain of the decision attribute Health is equal to $\{0,1\}$. The decision attribute Health divides the set decision objects (i.e. readings) into two preference-ordered classes: $C l_{0}=\{0\}$ and $C l_{1}=\{1\}$. Thus, the unions of classes that should be approximated are:

- $C l_{0}^{\leq}$; i.e., the objects belonging to (at most) class $C l_{0}$,

- $C l_{1}^{\geq}$; i.e., the objects belonging to (at least) class $C l_{1}$.

The second analysis corresponds to Level 2 in Figure 4. In this case, both the criteria Pressure and Temperature are specified as gain-type. The domain of the decision attribute Health is now equal to $\{0,2\}$. The decision attribute Health divides the set decision objects into two preference-ordered classes: $C l_{0}=\{0\}$ and $C l_{2}=\{2\}$. Thus, the unions of classes that should be approximated are:

- $C l_{0}^{\leq}$; i.e., the objects belonging to (at most) class $C l_{0}$,

- $C l_{2}^{\geq}$; i.e., the objects belonging to (at least) class $C l_{2}$.

These unions of classes have been approximated using the decision tables and the equations given in Section 3.1. The result of approximation of decision tables relative to the 9 th range $T_{9}$ and the 30 th range $T_{30}$ along with the corresponding quality of the approximation and accuracy of the rough-set representation of classes of input data are summarised in Appendix B.

Table 4 shows the decision rules inferred from the decision tables relative ranges $T_{9}$ and $T_{30}$. This table indicates also the: (i) supporting objects (readings in this application case), which are the objects having a description that matches both the condition and the decision parts of the decision rules; and (ii) the relative strength, which is the number of positive examples covered (i.e. objects with a description that matches at least the condition part of the rule) by the rule divided by the number of all positive examples in the class. As shown in Table 4, all of the decision rules show a relative strength of $100 \%$. 
Table 3: Decision tables for ranges $T_{9}$ (a) and $T_{30}$ (b)

\begin{tabular}{|c|c|c|c|}
\hline$\#$ & Temperature & Pressure & Health \\
\hline 1 & 9 & 100 & 1 \\
2 & 9 & 103 & 1 \\
3 & 9 & 106 & 1 \\
4 & 9 & 109 & 1 \\
5 & 9 & 112 & 1 \\
6 & 9 & 115 & 0 \\
7 & 9 & 118 & 0 \\
8 & 9 & 121 & 0 \\
9 & 9 & 124 & 2 \\
10 & 9 & 127 & 2 \\
11 & 9 & 130 & 2 \\
12 & 9 & 133 & 2 \\
13 & 9 & 136 & 2 \\
14 & 9 & 139 & 2 \\
15 & 9 & 142 & 2 \\
16 & 9 & 145 & 2 \\
17 & 9 & 148 & 2 \\
18 & 9 & 151 & 2 \\
19 & 9 & 154 & 2 \\
20 & 9 & 157 & 2 \\
21 & 9 & 160 & 2 \\
22 & 9 & 163 & 2 \\
23 & 9 & 166 & 2 \\
24 & 9 & 169 & 2 \\
\hline & & & \\
& 9 & & \\
\hline
\end{tabular}

\begin{tabular}{|c|c|c|c|}
\hline$\#$ & Temperature & Pressure & Health \\
\hline 25 & 30 & 100 & 1 \\
26 & 30 & 103 & 1 \\
27 & 30 & 106 & 1 \\
28 & 30 & 109 & 1 \\
29 & 30 & 112 & 1 \\
30 & 30 & 115 & 1 \\
31 & 30 & 118 & 1 \\
32 & 30 & 121 & 1 \\
33 & 30 & 124 & 0 \\
34 & 30 & 127 & 0 \\
35 & 30 & 130 & 0 \\
36 & 30 & 133 & 2 \\
37 & 30 & 136 & 2 \\
38 & 30 & 139 & 2 \\
39 & 30 & 142 & 2 \\
40 & 30 & 145 & 2 \\
41 & 30 & 148 & 2 \\
42 & 30 & 151 & 2 \\
43 & 30 & 154 & 2 \\
44 & 30 & 157 & 2 \\
45 & 30 & 160 & 2 \\
46 & 30 & 163 & 2 \\
47 & 30 & 166 & 2 \\
48 & 30 & 169 & 2 \\
\hline
\end{tabular}

Table 4: Initial decision rules for pressure-oriented analysis for ranges $T_{9}$ and $T_{30}$

\begin{tabular}{|c|c|c|c|c|c|}
\hline Range & $\begin{array}{c}\text { Analysis } \\
\text { level }\end{array}$ & $\begin{array}{c}\text { Rule } \\
\#\end{array}$ & $\begin{array}{l}\text { Rule } \\
\text { description }\end{array}$ & $\begin{array}{l}\text { Supporting } \\
\text { objects }\end{array}$ & $\begin{array}{c}\text { Relative } \\
\text { strength }(\%)\end{array}$ \\
\hline \multirow[t]{4}{*}{$T_{9}$} & \multirow[t]{2}{*}{ Level 1} & 1 & If (Pressure $>115)$ then (Health=At most 0 ); & $6,7,8$ & 100 \\
\hline & & 2 & If (Pressure $\leq 112$ ) then (Health=At least 1 ); & $1,2,3,4,5$ & 100 \\
\hline & \multirow[t]{2}{*}{ Level 2} & 3 & If (Pressure $\leq 121$ ) then (Health=At most 0 ); & $6,7,8$ & 100 \\
\hline & & 4 & If (Pressure $>124)$ then (Health=At least 2 ); & $9,10,11,12,13,14,15,16,17,18,19,20,21,22,23,24$ & 100 \\
\hline \multirow[t]{4}{*}{$T_{30}$} & \multirow[t]{2}{*}{ Level 1} & 5 & If(Pressure $>124)$ then $($ Health $=$ At most 0$)$; & $33,34,35$ & 100 \\
\hline & & 6 & If (Pressure $\leq 121)$ then (Health=At least 1 ); & $25,26,27,28,29,30,31,32$ & 100 \\
\hline & \multirow[t]{2}{*}{ Level 2} & 7 & If (Pressure $<130)$ then (Health=At most 0 ); & $33,34,35$ & 100 \\
\hline & & 8 & If (Pressure $>133$ ) then (Health=At least 2 ); & $36,37,38,40,41,42,43,44,45,46,47,48$ & 100 \\
\hline
\end{tabular}

The generated decision rules are first validated through the reclassification strategy. The result of reclassification can be summarised through an $n \times n$ confusion matrix where $n$ is the number of decision classes. The intersection of a row and column indicates the number of original and possible assignments for the decision classes corresponding to the considered row and column. The confusion matrices relative to the range $T_{9}$ for analysis level 1 and analysis level 2 are shown in Table 5(a) and Table 5(b), respectively; and those relative to the range $T_{30}$ are shown in Table 5(c) (for analysis level 1) and Table 5(d) (for analysis level 2). All confusion matrices show a perfect match between the original assignments and those proposed by the system.

Table 5: Confusion matrices relative to initial decision rules for pressure-oriented analysis for ranges $T_{9}$ and $T_{30}$

\begin{tabular}{|c|cc|}
\hline \multirow{2}{*}{ Original } & Proposed & \\
\cline { 2 - 3 }$C l_{0}$ & $C l_{0}$ & $C l_{1}$ \\
$C l_{1}$ & $3 / 3$ & $0 / 0$ \\
& $0 / 0$ & $5 / 5$ \\
\hline
\end{tabular}

\begin{tabular}{|c|cc|}
\hline \multirow{2}{*}{ Original } & Proposed & \\
\cline { 2 - 3 }$C l_{0}$ & $C l_{0}$ & $C l_{1}$ \\
\hline$C l_{1}$ & $3 / 3$ & $0 / 0$ \\
& $0 / 0$ & $16 / 16$ \\
\hline
\end{tabular}

\begin{tabular}{|c|cc|}
\hline \multirow{2}{*}{ Original } & Proposed & \\
\cline { 2 - 3 }$C l_{0}$ & $C l_{1}$ \\
\hline$C l_{0}$ & $3 / 3$ & $0 / 0$ \\
$C l_{1}$ & $0 / 0$ & $8 / 8$ \\
\hline \multicolumn{2}{|c}{ (c) }
\end{tabular}

\begin{tabular}{|c|cc|}
\hline \multirow{2}{*}{ Original } & Proposed & \\
\cline { 2 - 3 }$C l_{0}$ & $C l_{1}$ \\
\hline$C l_{0}$ & $3 / 3$ & $0 / 0$ \\
$C l_{1}$ & $0 / 0$ & $13 / 13$ \\
\hline
\end{tabular}

Based on the discussion in Section 3.3, the initial set of decision rules need to be refined by: (i) removing redundant rules; and, (ii) adding the 'implicit' condition about the fixed criterion. The rules \#1 to \#4 in Table 4 are first replaced 
by the following three decision rules:

- If (Pressure $>115)$ and (Pressure $<124)$ then (Health=At most 0);

- If (Pressure $\leq 115)$ then $($ Health=At least 1$)$;

- If (Pressure $\geq 124)$ then (Health=At least 2).

Then, by adding the implicit condition about the fixed criterion, we obtain the following three decision rules for range $T_{9}$ :

- If (Temperature $\geq 9)$ and $($ Temperature $<12)$ and $($ Pressure $>115)$ and $($ Pressure $<124)$ then $($ Health=At most 0$)$;

- If (Temperature $\geq 9)$ and (Temperature $<12)$ and (Pressure $\leq 115)$ then (Health=At least 1$)$;

- If (Temperature $\geq 9)$ and (Temperature $<12)$ and (Pressure $\geq 124)$ then (Health=At least 2).

The application of the same transformation operations on the decision rules obtained from decision table relative to the range $T_{30}$ leads to the following decision rules:

- If (Temperature $\geq 30)$ and (Temperature $<33)$ and (Pressure $>124)$ and (Pressure $<130)$ then (Health=At most 0$)$;

- If (Temperature $\geq 30)$ and (Temperature $<33)$ and (Pressure $\leq 124)$ then (Health=At least 1 );

- If (Temperature $\geq 30$ ) and (Temperature $<33$ ) and (Pressure $\geq 130$ ) then (Health=At least 2).

\subsubsection{Temperature-oriented analysis}

The temperature-oriented analysis is based on a discretisation of the pressure criterion and then for each pressure value we define a decision table composed of two criteria corresponding to Temperature and Pressure, and one decision attribute corresponding to the tyre health. Because the values of Pressure criterion vary in the range [100,169], a collection of 35 initial decision tables have been constructed. Table 6 provides the decision tables corresponding to pressure in the ranges $P_{20}$ which covers the pressure values in $[120,121]$ and $P_{22}$ which covers the pressure values in $[122,123]$.

Two different analyses was conducted for each range: one uses the classes $C l_{0}$ and $C l_{1}$, and the other uses the classes $\mathrm{Cl}_{0}$ and $\mathrm{Cl}_{2}$. The first analysis corresponds to Level 3 in Figure 4. As indicated in Table 3 (in the fourth column), the criteria Pressure and Temperature are specified as cost-type and gain-type, respectively. In addition, the domain of the decision attribute Health is equal to $\{0,1\}$. The decision attribute Health divides the set decision objects (i.e. readings) into two preference-ordered classes: $C l_{0}=\{0\}$ and $C l_{1}=\{1\}$. Thus, the unions of classes that should be approximated are:

- $C l_{0}^{\leq}$, i.e., the objects belonging to (at most) class $C l_{0}$,

- $C l_{1}^{\geq}$, i.e., the objects belonging to (at least) class $C l_{1}$. 
The second analysis corresponds to Level 4 in Figure 4. In this case, both the criteria Pressure and Temperature are specified as gain-type and cost-type, respectively. The domain of the decision attribute Health is now equal to $\{0,2\}$. The decision attribute Health divides the set decision objects into two preference-ordered classes: $C l_{0}=\{0\}$ and $C l_{2}=\{2\}$. Thus, the unions of classes that should be approximated are:

- $C l_{0}^{\leq}$, i.e., the objects belonging to (at most) class $C l_{0}$,

- $C l_{2}^{\geq}$, i.e., the objects belonging to (at least) class $C l_{2}$.

These unions of classes have been approximated using the decision tables and the equations given in Section 3.1. The result of approximation of the decision tables relative to ranges $P_{20}$ and $P_{22}$ along with the corresponding quality of the approximation and accuracy of the rough-set representation of classes of input data are summarised in Appendix B.

Table 7 (which has the same description as Table 4 as presented in Section 5.3.1) shows the decision rules inferred from decision tables relative to ranges $P_{20}$ and $P_{22}$. As shown in Table 7, all of the decision rules show a relative strength of $100 \%$. The generated decision rules were validated through the reclassification strategy. The confusion matrices relative to the range $P_{20}$ for analysis levels 3 and 4 are shown in Tables 8(a) and 8(b), respectively; and those relative to the range $P_{22}$ are shown in Tables 8(c) and 8(d), respectively. All of the confusion matrices show a perfect match between the original assignments and those proposed by the system.

Using the same transformation operations that we introduced in Section 3.3, decision rules \#9 to \#12 in Table 7 lead to the following three decision rules:

- If (Pressure $\geq 20)$ and (Pressure $<22)$ and (Temperature $>8$ ) and (Temperature $<22)$ then (Health=At most 0 );

- If (Pressure $\geq 20$ ) and (Pressure $<22$ ) and (Temperature $\geq 22$ ) then (Health=At least 1);

- If (Pressure $\geq 20$ ) and (Pressure $<22$ ) and (Temperature $\geq 8$ ) then (Health=At least 2).

Similarly, decision rules \#13 to \#16 in Table 7 lead to the following three decision rules:

- If (Pressure $\geq 22)$ and (Pressure $<24)$ and (Temperature $>10$ ) and (Temperature $<28)$ then (Health=At most 0 );

- If (Pressure $\geq 20$ ) and (Pressure $<24)$ and (Temperature $\geq 28$ ) then (Health=At least 1 );

- If (Pressure $\geq 22$ ) and (Pressure $<24)$ and (Temperature $\leq 10)$ then (Health=At least 2 ).

\section{Comparison and validation with large datasets}

This section presents a comparative study of the monitoring rules of DRSA, the threshold-based system and decision tree (Section 6.1). It also contains the evaluation of the proposed approach as compared to the threshold-based approach and decision tree (Section 6.2). Finally, a summary of the analysis is also presented (Section 6.3). 
Table 6: Decision tables for ranges $P_{20}$ (a) or $P_{22}$ (b)

\begin{tabular}{|c|c|c|c|}
\hline$\#$ & Pressure & Temperature & Health \\
\hline 1 & 120 & 0 & 2 \\
\hline 2 & 120 & 2 & 2 \\
\hline 3 & 120 & 4 & 2 \\
\hline 4 & 120 & 6 & 2 \\
\hline 5 & 120 & 8 & 0 \\
\hline 6 & 120 & 10 & 0 \\
\hline 7 & 120 & 12 & 0 \\
\hline 8 & 120 & 14 & 0 \\
\hline 9 & 120 & 16 & 0 \\
\hline 10 & 120 & 18 & 0 \\
\hline 11 & 120 & 20 & 0 \\
\hline 12 & 120 & 22 & 0 \\
\hline 13 & 120 & 24 & 1 \\
\hline 14 & 120 & 26 & 1 \\
\hline 15 & 120 & 28 & 1 \\
\hline 16 & 120 & 30 & 1 \\
\hline 17 & 120 & 32 & 1 \\
\hline 18 & 120 & 34 & 1 \\
\hline 19 & 120 & 36 & 1 \\
\hline 20 & 120 & 38 & 1 \\
\hline 21 & 120 & 40 & 1 \\
\hline 22 & 120 & 42 & 1 \\
\hline 23 & 120 & 44 & 1 \\
\hline 24 & 120 & 46 & 1 \\
\hline 25 & 120 & 48 & 1 \\
\hline 26 & 120 & 50 & 1 \\
\hline 27 & 120 & 52 & 1 \\
\hline 28 & 120 & 54 & 1 \\
\hline 29 & 120 & 56 & 1 \\
\hline 30 & 120 & 58 & 1 \\
\hline 31 & 120 & 60 & 1 \\
\hline 32 & 120 & 62 & 1 \\
\hline 33 & 120 & 64 & 1 \\
\hline 34 & 120 & 66 & 1 \\
\hline 35 & 120 & 68 & 1 \\
\hline 36 & 120 & 70 & 1 \\
\hline 37 & 120 & 72 & 1 \\
\hline 38 & 120 & 74 & 1 \\
\hline 39 & 120 & 76 & 1 \\
\hline 40 & 120 & 78 & 1 \\
\hline 41 & 120 & 80 & 1 \\
\hline 42 & 120 & 82 & 1 \\
\hline 43 & 120 & 84 & 1 \\
\hline 44 & 120 & 86 & 1 \\
\hline 45 & 120 & 88 & 1 \\
\hline 46 & 120 & 90 & 1 \\
\hline 47 & 120 & 92 & 1 \\
\hline 48 & 120 & 94 & 1 \\
\hline 49 & 120 & 96 & 1 \\
\hline 50 & 120 & 98 & 1 \\
\hline 51 & 120 & 100 & 1 \\
\hline
\end{tabular}

\begin{tabular}{|c|c|c|c|}
\hline$\#$ & Pressure & Temperature & Health \\
\hline 52 & 122 & 0 & 2 \\
\hline 53 & 122 & 2 & 2 \\
\hline 54 & 122 & 4 & 2 \\
\hline 55 & 122 & 6 & 2 \\
\hline 56 & 122 & 8 & 2 \\
\hline 57 & 122 & 10 & 2 \\
\hline 58 & 122 & 12 & 0 \\
\hline 59 & 122 & 14 & 0 \\
\hline 60 & 122 & 16 & 0 \\
\hline 61 & 122 & 18 & 0 \\
\hline 62 & 122 & 20 & 0 \\
\hline 63 & 122 & 22 & 0 \\
\hline 64 & 122 & 24 & 0 \\
\hline 65 & 122 & 26 & 0 \\
\hline 66 & 122 & 28 & 1 \\
\hline 67 & 122 & 30 & 1 \\
\hline 68 & 122 & 32 & 1 \\
\hline 69 & 122 & 34 & 1 \\
\hline 70 & 122 & 36 & 1 \\
\hline 71 & 122 & 38 & 1 \\
\hline 72 & 122 & 40 & 1 \\
\hline 73 & 122 & 42 & 1 \\
\hline 74 & 122 & 44 & 1 \\
\hline 75 & 122 & 46 & 1 \\
\hline 76 & 122 & 48 & 1 \\
\hline 77 & 122 & 50 & 1 \\
\hline 78 & 122 & 52 & 1 \\
\hline 79 & 122 & 54 & 1 \\
\hline 80 & 122 & 56 & 1 \\
\hline 81 & 122 & 58 & 1 \\
\hline 82 & 122 & 60 & 1 \\
\hline 83 & 122 & 62 & 1 \\
\hline 84 & 122 & 64 & 1 \\
\hline 85 & 122 & 66 & 1 \\
\hline 86 & 122 & 68 & 1 \\
\hline 87 & 122 & 70 & 1 \\
\hline 88 & 122 & 72 & 1 \\
\hline 89 & 122 & 74 & 1 \\
\hline 90 & 122 & 76 & 1 \\
\hline 91 & 122 & 78 & 1 \\
\hline 92 & 122 & 80 & 1 \\
\hline 93 & 122 & 82 & 1 \\
\hline 94 & 122 & 84 & 1 \\
\hline 95 & 122 & 86 & 1 \\
\hline 96 & 122 & 88 & 1 \\
\hline 97 & 122 & 90 & 1 \\
\hline 98 & 122 & 92 & 1 \\
\hline 99 & 122 & 94 & 1 \\
\hline 100 & 122 & 96 & 1 \\
\hline 101 & 122 & 98 & 1 \\
\hline 102 & 122 & 100 & 1 \\
\hline
\end{tabular}

(a)

(b)

Table 7: Initial decision rules for pressure-oriented analysis for ranges $P_{20}$ and $P_{22}$

\begin{tabular}{|c|c|c|c|c|c|}
\hline Range & $\begin{array}{c}\text { Analysis } \\
\text { level }\end{array}$ & $\begin{array}{c}\text { Rule } \\
\#\end{array}$ & $\begin{array}{l}\text { Rule } \\
\text { description }\end{array}$ & $\begin{array}{l}\text { Supporting } \\
\text { objects }\end{array}$ & $\begin{array}{c}\text { Relative } \\
\text { strength (\%) }\end{array}$ \\
\hline \multirow[t]{4}{*}{$P_{20}$} & \multirow[t]{2}{*}{ Level 3} & 9 & If (Temperature $\leq 22)$ then (Health=At most 0 ); & $5,6,7,8,9,10,11,12$ & 100 \\
\hline & & 10 & If (Temperature $\geq 24)$ then (Health=At least 1$)$ & $\begin{array}{l}13,14,15,16,17,18,19,20,21,22,23,24,25,26,27,28, \\
29,30,31,32,33,34,35,36,37,38,39,40,41,42,43,44, \\
45,46,47,48,49,50,51\end{array}$ & 100 \\
\hline & \multirow[t]{2}{*}{ Level 4} & 11 & If (Temperature $\geq 8$ ) then (Health=At most 0 ); & $5,6,7,8,9,10,11,12$ & 100 \\
\hline & & 12 & If (Temperature $\leq 6)$ then (Health=At least 2 ); & $1,2,3,4$ & 100 \\
\hline \multirow[t]{4}{*}{$P_{22}$} & \multirow[t]{2}{*}{ Level 3} & 13 & If (Temperature $\leq 26)$ then (Health=At most 0 ); & $58,59,60,61,62,63,64,65$ & 100 \\
\hline & & 14 & If (Temperature $\geq 28$ ) then (Health=At least 1$)$ & $\begin{array}{l}66,67,68,69,70,71,72,73,74,75,76,77,78,79,80,81 \\
82,83,84,85,86,87,88,89,90,91,92,93,94,95,96,97, \\
98,99,100,101,102\end{array}$ & 100 \\
\hline & \multirow[t]{2}{*}{ Level 4} & 15 & If (Temperature $\geq 12$ ) then (Health=At most 0 ); & $58,59,60,61,62,63,64,65$ & 100 \\
\hline & & 16 & If (Temperature $\leq 10)$ then (Health=At least 2$)$ & $52,53,54,55,56,57$ & 100 \\
\hline
\end{tabular}


Table 8: Confusion matrices relative to initial decision rules for pressure-oriented analysis for ranges $P_{20}$ and $P_{22}$

\begin{tabular}{|c|cc|}
\hline \multirow{2}{*}{ Original } & Proposed & \\
\cline { 2 - 3 }$C l_{0}$ & $C l_{0}$ & $C l_{1}$ \\
\hline$C l_{1}$ & $0 / 8$ & $0 / 0$ \\
& $0 / 0$ & $39 / 39$ \\
\hline
\end{tabular}

(a)

\begin{tabular}{|c|cc|}
\hline \multirow{3}{*}{ Original } & Proposed & \\
\cline { 2 - 3 }$C l_{0}$ & $C l_{0}$ & $C l_{1}$ \\
\hline$C l_{1}$ & $8 / 8$ & $0 / 0$ \\
& $0 / 0$ & $37 / 37$ \\
\hline
\end{tabular}

(c)

\begin{tabular}{|c|cc|}
\hline \multirow{2}{*}{ Original } & Proposed & \\
\cline { 2 - 3 }$C l_{0}$ & $C l_{0}$ & $C l_{1}$ \\
$C l_{1}$ & $0 / 8$ & $0 / 0$ \\
& $0 / 4$ \\
\hline
\end{tabular}

(b)

\begin{tabular}{|c|cc|}
\hline \multirow{3}{*}{ Original } & Proposed & \\
\cline { 2 - 3 }$C l_{0}$ & $C l_{0}$ & $C l_{1}$ \\
$C l_{1}$ & $8 / 8$ & $0 / 0$ \\
& $0 / 0$ & $6 / 6$ \\
\hline
\end{tabular}

\subsection{Comparative study}

We compared the monitoring rules obtained through DRSA with the threshold-based monitoring system initially adopted by the company. In the current system, the baseline for pressure is $120 \mathrm{PSI}$ and for temperature is $18^{\circ} \mathrm{C}$. Concerning pressure, the threshold-based monitoring rules are as follows:

1. If the pressure decreases by $20 \%$ below the baseline, then this will lead to low pressure, i.e., under-inflation.

2. If the pressure increases by $30 \%$ above the baseline, then this will lead to high pressure, i.e., over-inflation.

3. Anything within the above range is considered as a normal situation unless the temperature is above $85^{\circ} \mathrm{C}$.

The current system will detect a high-temperature fault when the temperature exceeds $85^{\circ} \mathrm{C}$. The above thresholdbased monitoring rules have been applied to the data in Tables 3 and 6 . The results of this application are summarised in Table 9. This table indicates the accuracy of the threshold-based monitoring rules. As this table shows, the accuracy of the pressure-oriented analysis levels is $30 \%$ and $50 \%$ while the accuracy for temperature-oriented analysis levels is $40 \%$ and $70 \%$.

Table 9: Accuracy values for threshold-based monitoring rules
\begin{tabular}{|c|c|c|}
\hline Analysis level & Decision table & Accuracy $(\%)$ \\
\hline Pressure-oriented & $T_{9}$ & 30 \\
\cline { 2 - 3 } & $T_{30}$ & 50 \\
\hline Temperature oriented & $P_{20}$ & 40 \\
\cline { 2 - 3 } & $P_{22}$ & 70 \\
\hline
\end{tabular}

We also compared the proposed system with decision tree-based monitoring. The obtained decision trees for the data in Tables 3 and 6 are given in Figure 9. We note that the labels A, B and C in Figure 9 correspond to labels 0, 1 and 2 of the decision classes. This is because the software KNIME that has been used to construct the decision trees accepts only nominal values for the decision classes. These decision trees have been used to reclassify the input data. The results of this application are summarised in Table 10. As shown in this table, the decision tree achieved a perfect reclassification for all input data. However, as discussed in the next subsection, the application of the decision tree to large datasets leads to insufficient results.

Table 10: Accuracy values for decision tree-based monitoring rules

\begin{tabular}{|c|c|c|}
\hline Analysis level & Decision table & Accuracy (\%) \\
\hline Pressure-oriented & $T_{9}$ & 100 \\
\cline { 2 - 3 } & $T_{30}$ & 100 \\
\hline Temperature oriented & $P_{20}$ & 100 \\
\cline { 2 - 3 } & $P_{22}$ & 100 \\
\hline
\end{tabular}




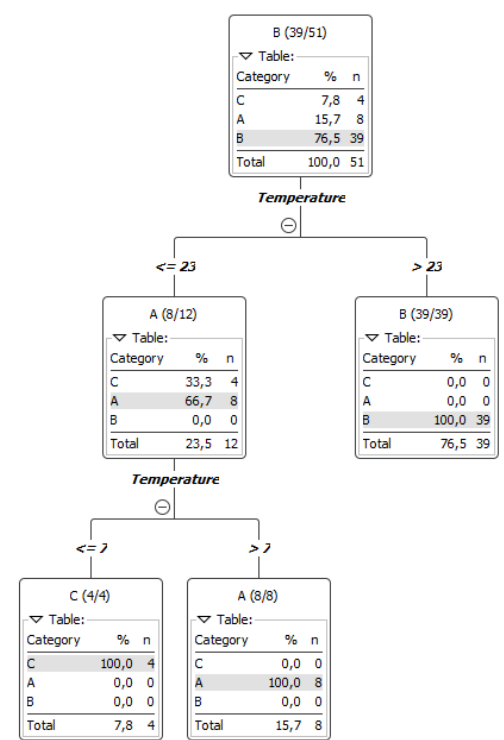

(a)

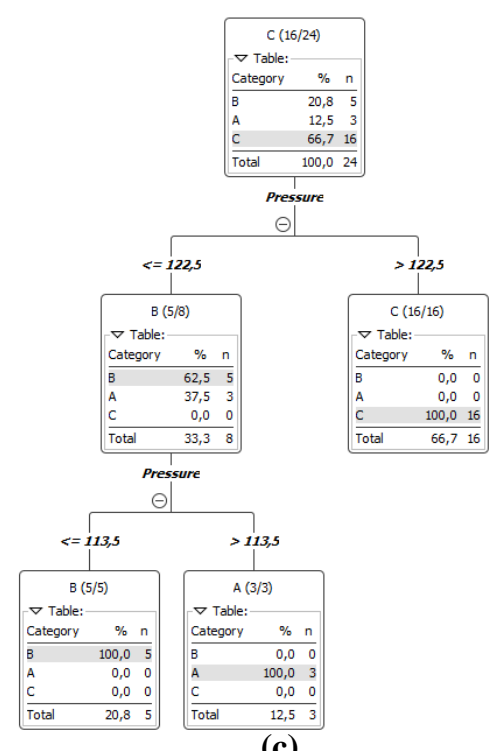

(c)

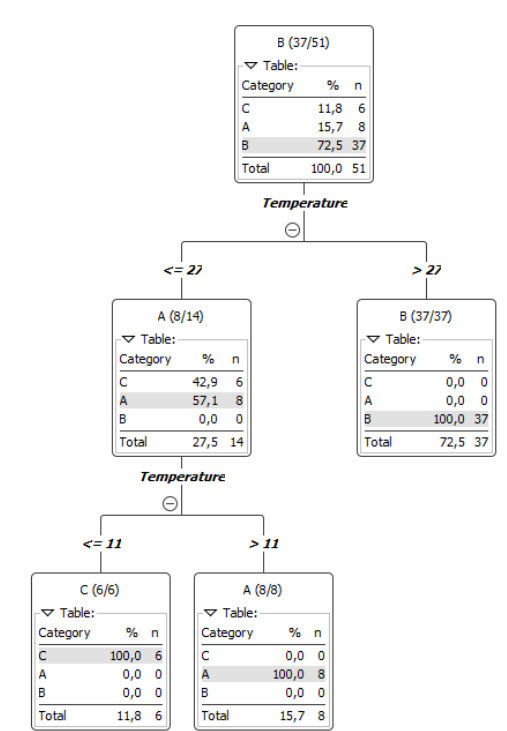

(b)

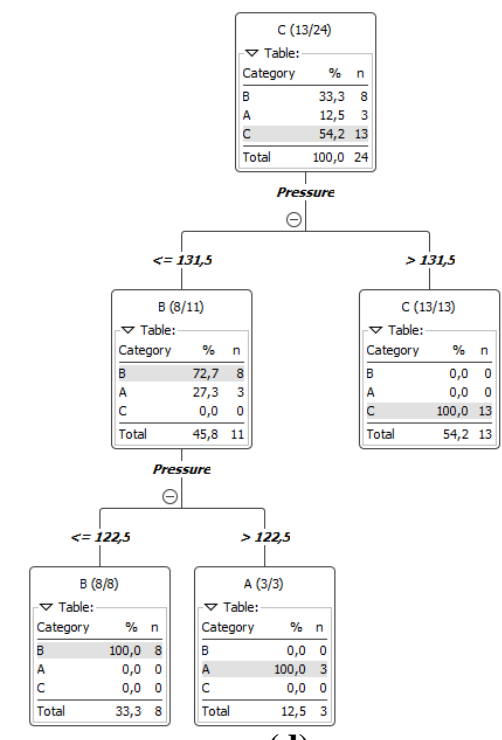

(d)

Figure 9: Decision trees relative to decision tables corresponding to ranges: $P_{20}$ (a), $P_{22}$ (b), $T_{9}$ (c) and $T_{30}$ (d)

\subsection{Validation with large datasets}

The decision rules that were obtained have been validated using datasets from two separate coaches. The description of these datasets is given in Table 11. The health of the considered observations in this table is based on the information reported by the drivers. These observations have been classified by all decision rules. As shown in the confusion matrices in Table 12, there is a close agreement between the health of the tyres as reported by the drivers and the health of the tyres as computed by the decision rules.

For comparison purposes, we applied the threshold-based and decision tree-based monitoring rules to the two large datasets given in Table 11. The results of this additional comparative study are summarised in Table 13. The latter 
Table 11: Large testing datasets

\begin{tabular}{|c|ccc|c|c|}
\hline Dataset & Health & & & Reading & \\
\cline { 2 - 4 }$\#$ & 1 & 0 & 2 & errors & Total \\
\hline 1 & 4393 & 6303 & 1328 & 20 & 12044 \\
2 & 1693 & 6231 & 4580 & 15 & 12519 \\
\hline
\end{tabular}

Table 12: Confusion matrices relative to large testing datasets

\begin{tabular}{|c|ccc|}
\hline \multirow{3}{*}{ Original } & \multicolumn{3}{|c|}{ Proposed } \\
\cline { 2 - 4 }$C l_{1}$ & $4393 / 4393$ & $C l_{0}$ & $C l_{2}$ \\
\hline$C l_{0}$ & $0 / 0$ & $6303 / 6303$ & $0 / 0$ \\
$C l_{2}$ & $0 / 0$ & $0 / 0$ & $1328 / 1328$ \\
\hline \multicolumn{4}{|c}{ Dataset \#1 }
\end{tabular}

\begin{tabular}{|c|c|c|c|}
\hline \multirow[b]{2}{*}{ Original } & \multicolumn{3}{|c|}{ Proposed } \\
\hline & $C l_{1}$ & $C l_{0}$ & $\mathrm{Cl}_{2}$ \\
\hline$C l_{1}$ & $1693 / 1693$ & $0 / 0$ & $0 / 0$ \\
\hline$C l_{0}$ & $0 / 0$ & $6231 / 6231$ & $0 / 0$ \\
\hline $\mathrm{Cl}_{2}$ & $0 / 0$ & $0 / 0$ & $4580 / 4580$ \\
\hline
\end{tabular}

shows that threshold-based monitoring achieves low accuracy. The decision tree performed better than threshold-based system; however, it under performed the proposed DRSA approach.

Table 13: Accuracy values for threshold-based and decision tree-based monitoring

\begin{tabular}{|c|c|c|}
\hline Monitoring rules & Dataset & Accuracy (\%) \\
\hline \multirow{2}{*}{ Thresholds } & $\# 1$ & 19 \\
\cline { 2 - 3 } & $\# 2$ & 22 \\
\hline \multirow{2}{*}{ Decision tree } & $\# 1$ & 62 \\
\cline { 2 - 3 } & $\# 2$ & 57 \\
\hline
\end{tabular}

To further validate the obtained results, a series of well-known non-parametric statistics are used to compare the initial and computed tyre health values. The statistics considered are Kendall's tau, Spearman's rho, and the Unweighted and Weighted Cohen's kappa. The result of this statistical comparison is given in Table 14. The figures in Table 14 indicate close agreement levels between the real tyre health values and those computed by the generated monitoring rules. In fact, the values for Kendall's tau and Spearman's rho are higher than 80\%, which indicates a high agreement level. This result is also confirmed by unweighted and weighted Cohen's kappa where the agreement levels are higher than $60 \%$. This means there is a strong agreement level between real and computed tyre health values. The low values for the MAE further confirm the previous results.

Likewise, the figures in Table 14 show that the threshold-based or decision tree-based monitoring rules have a low to very low agreement levels between real health values and those computed by these methods.

\begin{tabular}{|l|cc|cc|cc|}
\hline \multicolumn{9}{|c|}{ Table 14: Statistical analysis } \\
\hline Method & DRSA & & Thresholds & Decision tree \\
\cline { 2 - 7 } Statistics & Dataset 1 & Dataset 2 & Dataset 1 & Dataset 2 & Dataset 1 & Dataset 2 \\
\hline Kendall's tau & 0.8712 & 0.8236 & 0.2395 & 0.3427 & 0.5734 & 0.4679 \\
Spearman's rho & 0.8534 & 0.892 & 0.3193 & 0.3591 & 0.6224 & 0.5064 \\
Unweighted Cohen's kappa & 0.7616 & 0.7938 & 0.1902 & 0.2472 & 0.2869 & 0.3381 \\
Weighted Cohen's kappa & 0.8361 & 0.7777 & 0.2014 & 0.1598 & 0.3499 & 0.4609 \\
\hline
\end{tabular}

\subsection{Summary of the comparative analysis}

In this comparative study, the proposed monitoring approach achieved better results than the threshold or decision tree-based approaches. This can be explained by the fact that these approaches fail to take into account the complex relationship between pressure and temperature variables. Also, neither threshold nor decision tree-based monitoring approach takes into account the ordinal relationship between the different decision classes. Although the decision 
tree-based monitoring system leads to perfect re-classification with the learning datasets, it fails when applied to large datasets. This is because the decision tree ignores the preference direction that characterises the criteria pressure and temperature.

Finally, it is important to mention that the proposed approach achieves $100 \%$ accuracy with no false positives or false negatives (as indicated in Table 12). Although that in the considered application learning and testings datasets have been randomly collected, one should pay attention to the possible overfitting of the proposed model. Some additional analysis have confirmed the results reported in this paper but a more intensive analysis (using other datasets from other coaches) should considered in the future.

\section{Conclusion and future research}

In this paper, an innovative decision rule-based approach for tyre monitoring is proposed. This approach relies on the DRSA, which is a well-known multicriteria classification and preference learning method. The proposed approach resolves the problem of false alarms that characterise the existing threshold-based tyre monitoring systems. This problem is caused by the complex relationship between tyre pressure and temperature, and is also due to sensors measurement. The problem of complex pressure/temperature relationship was resolved by fixing one of the parameters and generating the decision rules based on the other parameter combined with a discretisation of the values of the fixed parameter. Based on these solutions, an analysis strategy composed of two parallel analysis levels, namely pressure-oriented analysis and temperature-oriented analysis, was developed. The working principle of these analysis levels is to discretise the fixed parameter values into set of ordered ranges and to construct a decision table for each range, and then use the DRSA to infer a collection of decision rules.

The generated decision rules need to be validated and refined by adding the 'implicit' condition relative to the fixed parameter. The use of reclassification validation technique showed a very high degree of accuracy. The validated decision rules are used to develop and implement a classifier to monitor the health of the tyres. The classifier has been enhanced with several meta-rules, which enable the system to function even when one of the sensors is down, to detect the divergence between pressure-based and temperature-based decision rules, and to alert the driver when the sensors are broken.

The proposed approach has been validated and implemented within an important travelling company that operates in the South of England. Real-world tests show that the proposed approach considerably improved the tyre monitoring system and led to a substantial reduction of false alarms. At this point, it is important to mention that the proposed approach is generic enough, and can be used with other transport companies and different tyres type.

An advanced test phase is required. This intends intensive and large scale real-world tests of the developed system. There is also a need to design appropriate algorithms to exploit the large historical data to predict the tyres' health. The use of other monitoring criteria—such as speed, ambient temperature, road texture, road condition and load of the vehicle—should also be investigated. 


\section{References}

Blaszczyński, J., Greco, S., \& Slowiński, R. (2007). Multi-criteria classification - a new scheme for application of dominance-based decision rules. European Journal of Operational Research, 181, 1030-44.

Blaszczyński, J., Greco, S., \& Słowiński, R. (2012). Inductive discovery of laws using monotonic rules. Engineering Applications of Artificial Intelligence, 25, 284-94.

Błaszczyński, J., Słowiński, R., \& Szelg, M. (2010). Learnability in rough set approaches. In M. Szczuka, M. Kryszkiewicz, S. Ramanna, R. Jensen, \& Q. Hu (Eds.), Proceedings of The 7th International Conference on Rough Sets and Current Trends in Computing (RSCTC 2010) (pp. 402-11). Berlin, Heidelberg: Springer Berlin Heidelberg.

Błaszczyński, J., Słowiński, R., \& Szelạg, M. M. (2011). Sequential covering rule induction algorithm for variable consistency rough set approaches. Information Sciences, 181, 987-1002.

Chai, J., Liu, J., \& Xu, Z. (2013). A rule-based group decision model for warehouse evaluation under interval-valued intuitionistic fuzzy environments. Expert Systems with Applications, 40, 1959-70.

Chakhar, S., Ishizaka, A., Labib, A., \& Saad, I. (2016). Dominance-based rough set approach for group decisions. European Journal of Operational Research, 251, 206-24.

Chakhar, S., \& Saad, I. (2012). Dominance-based rough set approach for groups in multicriteria classification. Decision Support Systems, 54, 372-80.

Coppo, F., Pepe, G., Roveri, N., \& Carcaterra, A. (2017). A multisensing setup for the intelligent tire monitoring. Sensors, 17. Article \#576.

Garcia-Pozuelo, D., Olatunbosun, O., Yunta, J., Yang, X., \& Diaz, V. (2017). A novel strain-based method to estimate tire conditions using fuzzy logic for intelligent tires. Sensors, 17. Article \#874.

Greco, S., Matarazzo, B., \& Slowiński, R. (1999). The use of rough sets and fuzzy sets in MCDM. In T. Gal, T. Hanne, \& T. Stewart (Eds.), Advances in Multiple Criteria Decision Making (pp. 14.1-14.59). Dordrecht, Boston: Kluwer Academic Publishers.

Greco, S., Matarazzo, B., \& Słowiński, R. (2001). Rough sets theory for multicriteria decision analysis. European Journal of Operational Research, 129, 1-47.

Greco, S., Matarazzo, B., \& Słowiński, R. (2002). Multicriteria classification. In W. Kloesgen, \& J. Zytkow (Eds.), Handbook of Data Mining and Knowledge Discovery (pp. 318-27). New York: Oxford University Press.

Greco, S., Matarazzo, B., \& Słowiński, R. (2008). Dominance-based rough set approach to interactive multiobjective optimization. In J. Branke, K. Deb, K. Miettinen, \& R. Słowiński (Eds.), Multiobjective Optimization: Interactive and Evolutionary Approaches (pp. 121-55). Berlin, Heidelberg: Springer Berlin Heidelberg. 
Hasan, N., Arif, A., \& Pervez, U. (2011). Tire pressure monitoring system with wireless communication. In Proceedings of The 24th Canadian Conference on Electrical and Computer Engineering (CCECE 2011) (pp. 99-101). Niagara Falls, ON, Canada: IEEE.

Hu, Q., Chakhar, S., Siraj, S., \& Labib, A. (2017). Spare parts classification in industrial manufacturing using the dominance-based rough set approach. European Journal of Operational Research, 262, 1136-63.

Karhe, R., \& Patil, P. (2016). Intelligent tire pressure monitoring system based on android. International Journal of Science, Engineering and Technology Research, 5, 2305-8.

Kubba, A., \& Jiang, K. (2014). A comprehensive study on technologies of tyre monitoring systems and possible energy solutions. Sensors, 14, 10306-45.

Li, S., Tian, J., Zu, L., \& Yang, Z. (2010). Fuzzy comprehensive evaluation of tyre safety status based on analytic hierarchy process. In Proceedings of The 29th Chinese Control Conference (CCC 2010) (pp. 5414-20). Beijing, China: IEEE.

Liou, J., Tang, C.-H., Yeh, W.-C., \& Tsai, C.-Y. (2011). A decision rules approach for improvement of airport service quality. Expert Systems with Applications, 38, 13723-30.

Marton, Z., Fodor, D., Enisz, K., \& Nagy, K. (2014). Frequency analysis based tire pressure monitoring. In Proceedings of The IEEE International Electric Vehicle Conference (IEVC 2014) (pp. 1-5). Florence, Italy: IEEE.

Na, D., Shin, S., Lee, S., \& Lim, Y. (2016). A development of the validation system of indirect TPMS. In Proceedings of The International Congress of Automotive and Transport Engineering (CONAT 2016) (pp. 35-42). Brasov, Romania: Transilvania University Press.

Na, D., Shin, S., Lee, S., \& Lim, Y. (2017). A development of the validation system of indirect TPMS. Romanian Journal of Automotive Engineering, 23, 19-26.

Pawlak, Z. (1982). Rough sets. International Journal of Information \& Computer Sciences, 11, 341-56.

Pawlak, Z. (1991). Rough set. Theoretical aspects of reasoning about data. Dordrecht: Kluwer Academic Publishers.

Peters, G., \& Poon, S. (2011). Analyzing IT business values-a dominance based rough sets approach perspective. Expert Systems with Applications, 38, 11120-8.

Polkowski, L., \& Artiemjew, P. (2015). Learning data classification: Classifiers in general and in decision systems. In Granular Computing in Decision Approximation: An Application of Rough Mereology (pp. 33-62). Cham, Switzerland: Springer International Publishing volume 77 of Intelligent Systems Reference Library.

Premarsha, B. (2016). Survey on tire pressure monitoring system. International Journal of Engineering and Technical Research, 5, 120-1. 
Royal Automobile Club (2016). Tyre wear-accident statistics. http: / /www . rac.co.uk/my-rac/car-tips / wheel-tyres/tyres/accidents-and-statistics. Accessed 27 March 2018.

Saad, I., \& Chakhar, S. (2012). Multi-criteria methodology based on majority principle for collective identification of firm's valuable knowledge. Knowledge Management Research \& Practice, 10, 380-91.

Saaty, T. (1980). The Analytic Hierarchy Process. New York: McGraw-Hill.

Słowiński, R., Greco, S., \& Matarazzo, B. (2002). Rough set analysis of preference-ordered data. In J. Alpigini, J. Peters, A. Skowron, \& N. Zhong (Eds.), Rough Sets and Current Trends in Computing (pp. 44-59). Berlin, Heidelberg: Springer Berlin Heidelberg volume 2475 of Lecture Notes in Artificial Intelligence.

Słowiński, R., Greco, S., \& Matarazzo, B. (2012). Rough sets in decision making. In R. Meyers (Ed.), Computational Complexity (pp. 2727-60). New York: Springer New York.

Stefanowski, J., \& Vanderpooten, D. (2001). Induction of decision rules in classification and discovery-oriented perspectives. International Journal of Intelligent Systems, 16, 13-27.

Svensson, O., Thelin, S., Byttner, S., \& Fan, Y. (2017). Indirect tire monitoring system - machine learning approach. IOP Conference Series: Materials Science and Engineering, 252, 1-8.

Vishnoi, A., Rani, S., Singhal, D., Singh, A., \& Shinghal, K. (2015). Tire pressure monitoring system using wireless communication. International Journal of Scientific Research and Management Studies, 2, 105-11.

\section{AppendixA. Overview of the Dominance-based Rough Set Approach}

The DRSA has been proposed in (Greco et al., 2001, 2002; Słowiński et al., 2002, 2012) to overcome the shortcomings of the conventional Rough Sets Theory (RST) (Pawlak, 1982, 1991) in multicriteria classification by allowing preference-oriented attributes and is intended for use where decision classes are defined in an ordinal way. The basic idea of DRSA is to replace the indiscernibility relation used in the classical RST with the dominance relation, which is more appropriate for multicriteria classification. The DRSA has been successfully applied in different real-world decision problems, including risk assessment (Chakhar \& Saad, 2012), knowledge management (Saad \& Chakhar, 2012), service improvement (Liou et al., 2011), bankruptcy risk evaluation (Greco et al., 2002), supply chain management (Chai et al., 2013), supplier selection, airport service quality (Liou et al., 2011), product mix (Greco et al., 2008), performance of cooperations and business values (Peters \& Poon, 2011) and spare parts management (Hu et al., 2017). A brief overview of the DRSA is presented in this appendix.

\section{AppendixA.1. Basic definitions}

The information regarding the decision objects is often structured in a 4-tuple information table $\mathbf{S}=\langle U, Q, V, f\rangle$, where $U$ is a non-empty finite set of objects and $Q$ is a non-empty finite set of attributes such that $q: U \rightarrow V_{q}$ for every $q \in Q$. The $V_{q}$ is the domain of attribute $q, V=\bigcap_{q \in Q} V_{q}$, and $f: U \times Q \rightarrow V$ is the information function 
defined such that $f(x, q) \in V_{q}$ for each attribute $q$ and object $x \in U$. The set $Q$ is often divided into a subset $F \neq \emptyset$ of condition attributes and a subset $D \neq \emptyset$ of decision attributes, such that $F \cup D=Q$ and $F \cap D=\emptyset$. In this case, $\mathbf{S}$ is called a decision table. The domains of the condition attributes are supposed to be ordered according to a decreasing or increasing preference. These attributes are called criteria. The proponents of the DRSA assume that the preference is increasing with $f(\cdot, q)$ for every $q \in F$. They also assume that the set of decision attributes $D=\{E\}$ is a singleton. The unique decision attribute $E$ makes a partition of $U$ into a finite number of preference-ordered decision classes $\mathbf{C l}=\left\{C l_{t}, t \in T\right\}, T=\{0, \cdots, n\}$, such that each $x \in U$ belongs to one and only one class.

\section{AppendixA.2. Approximation}

In DRSA the represented knowledge is a collection of upward unions $C l_{t}^{\geq}$and downward unions $C l_{t}^{\leq}$of classes, defined as follows:

$$
C l_{\bar{t}}^{\geq}=\bigcup_{s \geq t} C l_{s}, C l_{t}^{\leq}=\bigcup_{s \leq t} C l_{s} .
$$

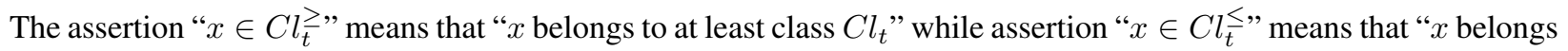
to at most class $C l_{t}$ ". The basic idea of DRSA is to replace the indiscernibility relation used in the conventional RST with a dominance relation. Let $P \subseteq C$ be a subset of condition criteria. The dominance relation $\Delta_{P}$ associated with $P$ is defined for each pair of objects $x$ and $y$, as follows: $x \Delta_{P} y \Leftrightarrow f(x, q) \succeq f(y, q), \forall q \in P$. In this definition, the symbol “ $\succeq$ ” should be replaced with “ $\preceq$ ” for criteria that are ordered according to decreasing preferences. To each object $x \in U$, we associate two sets: (i) the $P$-dominating set $\Delta_{P}^{+}(x)=\left\{y \in U: y \Delta_{P} x\right\}$ containing the objects that dominate $x$; and, (ii) the $P$-dominated set $\Delta_{P}^{-}(x)=\left\{y \in U: x \Delta_{P} y\right\}$ containing the objects dominated by $x$.

Then, the $P$-lower and $P$-upper approximations of $C l_{t}^{\geq}$with respect to $P$ are defined as follows:

- $\underline{P}\left(C l_{t}^{\geq}\right)=\left\{x \in U: \Delta_{P}^{+}(x) \subseteq C l_{t}^{\geq}\right\}$,

- $\bar{P}\left(C l_{\bar{t}}^{\geq}\right)=\left\{x \in U: \Delta_{P}^{-}(x) \cap C l_{t}^{\geq} \neq \emptyset\right\}$.

Analogously, the $P$-lower and $P$-upper approximations of $C l_{t} \leq$ with respect to $P$ are defined as follows:

- $\underline{P}\left(C l_{t}^{\leq}\right)=\left\{x \in U: \Delta_{P}^{-}(x) \subseteq C l_{t}^{\leq}\right\}$,

- $\bar{P}\left(C l_{t}^{\leq}\right)=\left\{x \in U: \Delta_{P}^{+}(x) \cap C l_{t}^{\leq} \neq \emptyset\right\}$.

The lower approximations group the objects that certainly belong to class unions $C l_{t}^{\geq}$(resp. $C l_{t}^{\leq}$). The upper approximations group the objects that could belong to $C l_{t}^{\geq}$(resp. $\left.C l_{t}^{\leq}\right)$.

The $P$-boundaries of $C l_{t}^{\geq}$and $C l_{t}^{\leq}$are defined as follows:

- $B n_{P}\left(C l_{t}^{\geq}\right)=\bar{P}\left(C l_{t}^{\geq}\right)-\underline{P}\left(C l_{t}^{\geq}\right)$,

- $B n_{P}\left(C l_{t}^{\leq}\right)=\bar{P}\left(C l_{t}^{\leq}\right)-\underline{P}\left(C l_{t}^{\leq}\right)$.

The boundaries group objects that can neither be ruled in nor out as members of class $C l_{t}$.

The quality of approximation of a partition $\mathbf{C l}$ by means of a set of criteria $P$ is defined as the ratio of all $P$ correctly classified objects to all objects in the system. The accuracy of the rough-set representation of unions of 
classes is computed as the ratio between the number of objects in the lower approximation and the number of objects in the upper approximation. In addition to these measures, the authors in Błaszczyński et al. (2010) introduce two additional measures to estimate the attainable predictive accuracy of a rough-set-based classifier. The first measure, called $\lambda$, estimates the attainable percentage of correctly classified objects of a classifier. The second measure, called $\delta$, estimates the attainable mean absolute error (MEA) of a classifier, which is defined as the mean absolute difference between the index of the class to which an object is assigned by a classifier and the index of the class to which the object belongs. The $\lambda$ and $\delta$ measures are only useful if the quality of approximation is low or even equal to zero. However, these measures have not been considered in the case study given in Section 5 because the quality of approximation on the learning dataset is equal to 1 .

The DRSA defines two concepts that may indicate some information about the importance of the criteria: the reduct and the core. A reduct is a minimal subset of criteria which can, by itself, fully characterise the knowledge in the decision table. The reduct of the decision table is not unique: there may be many subsets of criteria which preserve the equivalence classes. The set of attributes which is common to all reducts is called the core. Therefore, they are the criteria that cannot be removed from the decision table without causing the collapse of the equivalence classes. More information on these concepts is available in Greco et al. (2001) and Greco et al. (2002).

\section{AppendixA.3. Induction of decision rules}

A decision table may be seen as a set of 'if-then' decision rules. The condition part specifies the values assumed by one or more criteria, and the decision part specifies an assignment to one or more decision classes. The most popular rule induction algorithm for the DRSA is DOMLEM Greco et al. (2002), which generates a minimal set of rules. We also note the existence of a more recent rule induction algorithm, namely VC-DomLEM, proposed by Błaszczyński et al. (2011) and supersedes DOMLEM algorithm. Both algorithms follow a classical greedy scheme which produces a local covering of each decision concept, i.e., it covers all examples from the given approximation using a minimal set of rules.

Three types of decision rules may be considered in DRSA: (i) certain rules generated from the lower approximations; (ii) possible rules generated from the upper approximations; and (iii) approximate rules generated from the boundary regions. The general structures of certain decision rules are as follows:

\section{IF condition(s), THEN At Most $C l_{t}$ \\ IF condition(s), THEN At Least $C l_{t}$}

The decision part of a certain rule takes the form of an assignment to at most class unions or at least class unions. The general structures of possible decision rules are as follows:

IF condition(s), THEN Possibly At Most $C l_{t}$

IF condition(s), THEN Possibly At Least $C l_{t}$

In this case, the decision part specifies a possible assignment to at most class unions or at least class unions. Finally, the general structure of approximate rules is as follows: 
IF conditions on criteria values, THEN Belongs to $C l_{s} \cup C l_{s+1} \cup \cdots \cup C l_{t}$

Here, the decision part is defined as the union of several decision classes.

Decision rules are judged by their quality on the basis of the learning (or training) set, and by how they classify new unseen objects (Polkowski \& Artiemjew, 2015). Several measures have been proposed to evaluate the performance of decision rules. An object supports a decision rule if the description of the object matches both the condition and the decision parts of this rule. The support of a rule is the number of objects supporting the rule. A decision rule covers an object if the description of the object matches at least the condition part of the rule. The coverage is the number of the objects covered by the rule. The strength of a rule is the number of positive examples covered by the rule. The relative strength is the number of positive examples covered by the rule divided by the number of all positive examples in the union of classes. The confidence level (some authors call it consistency, or the certainty factor, or the precision) is defined as the number of positive examples covered by the rule, divided by the number of examples covered by the rule. For more information and for the formal definitions of all these concepts, see Polkowski \& Artiemjew (2015) and Stefanowski \& Vanderpooten (2001). Note that if the consequence is univocal (i.e. contains only one decision), then the rule is exact, otherwise it is approximate.

Finally, we should note that a given decision object may be covered by one or more decision rules, or may not be covered by any rule, in other situations. This issue has been discussed in detail in Blaszczyński et al. (2007), where the authors proposed different solutions to classify an objects using decision rules in one of three possible situations: it is covered by (i) no rule, (ii) exactly one rule, (iii) several rules. 


\section{AppendixB. Result of approximation}

Table B.15: Result of approximation for pressure-oriented analysis with respect to ranges $P_{20}$ and $P_{22}$

\begin{tabular}{|c|c|c|c|c|c|}
\hline Range & $\begin{array}{c}\text { Analysis } \\
\text { level }\end{array}$ & $\begin{array}{l}\text { Class } \\
\text { union }\end{array}$ & Lower approximation & Upper approximation & Boundary \\
\hline \multirow[t]{4}{*}{$P_{20}$} & \multirow[t]{2}{*}{ Level 1} & At most0 & $5,6,7,8,9,10,11,12$ & $5,6,7,8,9,10,11,12$ & $\emptyset$ \\
\hline & & At least 1 & $\begin{array}{l}13,14,15,16,17,18,19,20,21,22,23,24,25,26,27,28 \\
29,30,31,32,33,34,35,36,37,38,39,40,41,42,43,44, \\
45,46,47,48,49,50,51\end{array}$ & $\begin{array}{l}13,14,15,16,17,18,19,20,21,22,23,24,25,26,27,28, \\
29,30,31,32,33,34,35,36,37,38,39,40,41,42,43,44, \\
45,46,47,48,49,50,51\end{array}$ & $\emptyset$ \\
\hline & \multirow[t]{2}{*}{ Level 2} & At most 0 & $5,6,7,8,9,10,11,12$ & $5,6,7,8,9,10,11,12$ & $\emptyset$ \\
\hline & & At least 2 & $1,2,3,4$ & $1,2,3,4$ & $\emptyset$ \\
\hline \multirow{4}{*}{$P_{22}$} & \multirow[t]{2}{*}{ Level 1} & At most 0 & $58,59,60,61,62,63,64,65$ & $58,59,60,61,62,63,64,65$ & $\emptyset$ \\
\hline & & At least 1 & $\begin{array}{l}66,67,68,69,70,71,72,73,74,75,76,77,78,79,80,81 \\
82,83,84,85,86,87,88,89,90,91,92,93,94,95,96,97 \\
98,99,100,101,102\end{array}$ & $\begin{array}{l}66,67,68,69,70,71,72,73,74,75,76,77,78,79,80,81, \\
82,83,84,85,86,87,88,89,90,91,92,93,94,95,96,97, \\
98,99,100,101,102\end{array}$ & $\emptyset$ \\
\hline & \multirow[t]{2}{*}{ Level 2} & At most 0 & $58,59,60,61,62,63,64,65$ & $58,59,60,61,62,63,64,65$ & $\emptyset$ \\
\hline & & At least 2 & $2,53,54,55,56,57$ & $2,53,54,55,56,57$ & $\emptyset$ \\
\hline
\end{tabular}

Table B.16: Quality of approximation and accuracy for pressure-oriented analysis with respect to ranges $P_{20}$ and $P_{22}$

\begin{tabular}{|c|c|c|c|c|c|c|}
\hline \multirow[t]{3}{*}{ Range } & \multicolumn{3}{|l|}{ Analysis Level 1} & \multicolumn{3}{|l|}{ Analysis Level 2} \\
\hline & \multirow{2}{*}{$\begin{array}{c}\text { Quality of } \\
\text { approximation }\end{array}$} & \multicolumn{2}{|l|}{ Accuracy } & \multirow{2}{*}{$\begin{array}{c}\text { Quality of } \\
\text { approximation }\end{array}$} & \multicolumn{2}{|l|}{ Accuracy } \\
\hline & & At most 0 & At least 1 & & At most 0 & At least 2 \\
\hline$P_{20}$ & 1 & 1 & 1 & 1 & 1 & 1 \\
\hline$P_{22}$ & 1 & 1 & 1 & 1 & 1 & 1 \\
\hline
\end{tabular}

Table B.17: Result of approximation for temperature-oriented analysis with respect to ranges $T_{9}$ and $T_{30}$

\begin{tabular}{|c|c|c|c|c|c|}
\hline Range & $\begin{array}{c}\text { Analysis } \\
\text { level }\end{array}$ & $\begin{array}{l}\text { Class } \\
\text { union }\end{array}$ & Lower approximation & Upper approximation & Boundary \\
\hline \multirow[t]{4}{*}{$T_{9}$} & \multirow[t]{2}{*}{ Level 1} & At most 0 & $6,7,8$ & $6,7,8$ & $\emptyset$ \\
\hline & & At least 1 & $1,2,3,4,5$ & $1,2,3,4,5$ & $\emptyset$ \\
\hline & \multirow[t]{2}{*}{ Level 2} & At most 0 & $6,7,8$ & $6,7,8$ & $\emptyset$ \\
\hline & & At least 2 & $9,10,11,12,13,14,15,16,17,18,19,20,21,22,23,24$ & $9,10,11,12,13,14,15,16,17,18,19,20,21,22,23,24$ & $\emptyset$ \\
\hline \multirow[t]{4}{*}{$T_{30}$} & \multirow[t]{2}{*}{ Level 1} & At most 0 & $33,34,35$ & $33,34,35$ & $\emptyset$ \\
\hline & & At least 1 & $25,26,27,28,29,30,31,32$ & $25,26,27,28,29,30,31,32$ & $\emptyset$ \\
\hline & \multirow[t]{2}{*}{ Level 2} & At most 0 & $33,34,35$ & $33,34,35$ & $\emptyset$ \\
\hline & & At least 2 & $36,37,38,40,41,42,43,44,45,46,47,48$ & $36,37,38,40,41,42,43,44,45,46,47,48$ & $\emptyset$ \\
\hline
\end{tabular}

Table B.18: Quality of approximation and accuracy for temperature-oriented analysis with respect to ranges $T_{9}$ and $T_{30}$

\begin{tabular}{|c|c|c|c|c|c|c|}
\hline \multirow[t]{3}{*}{ Range } & \multicolumn{3}{|l|}{ Analysis Level 1} & \multicolumn{3}{|l|}{ Analysis Level 2} \\
\hline & \multirow{2}{*}{$\begin{array}{c}\text { Quality of } \\
\text { approximation }\end{array}$} & \multicolumn{2}{|l|}{ Accuracy } & \multirow{2}{*}{$\begin{array}{c}\text { Quality of } \\
\text { approximation }\end{array}$} & \multicolumn{2}{|l|}{ Accuracy } \\
\hline & & At most 0 & At least 1 & & At most 0 & At least 2 \\
\hline$T_{9}$ & 1 & 1 & 1 & 1 & 1 & 1 \\
\hline$T_{30}$ & 1 & 1 & 1 & 1 & 1 & 1 \\
\hline
\end{tabular}

